Pacific Journal of Mathematics

MONS WII REAL POLLS AND ZR 


\title{
FUNCTIONS WITH REAL POLES AND ZEROS
}

\author{
R. REDHEFFER
}

Throughout this paper $\left\{\lambda_{n}\right\}$ is a real sequence with $\lambda_{n} \neq 0$ and $\lambda_{n} \leqq \lambda_{n+1},-\infty<n<\infty$. The counting function $A_{1}(u)$ is the number of $\lambda_{n}$ between 0 and $u$, counted negatively for negative $u$. Similarly $\mu_{n}$ is a real sequence with $\mu_{n} \neq 0$ and with counting function $\Lambda_{2}(u)$. In this summary (which corresponds to the case $p=1$ of the paper) we define

$$
F(z)=\Pi \frac{\left(1-z / \lambda_{n}\right) e^{z / \lambda_{n}}}{\left(1-z / \mu_{n}\right) e^{z / \mu_{n}}}
$$

by taking all factors for which $\lambda_{n}$ and $\mu_{n}$ lie on the interval $(-R, R)$ and then letting $R \rightarrow \infty$. Our objective is to obtain conditions on the growth of $F(z)$ from conditions on the function $\Lambda(u)=\Lambda_{1}(u)-\Lambda_{2}(u)$.

Denoting the even part of $\Lambda(u)$ by $\Lambda_{e}(u)$, we can state our first result as follows: Suppose $\Lambda(u)=O(u)$ and

$$
\lim _{r \rightarrow \infty}\left(\frac{\Lambda(u r)}{u r}-\frac{\Lambda(r / u)}{r / u}\right)=0
$$

for each $u \neq 0$. Then

$$
\log \left|F\left(r e^{i \theta}\right)\right|=\pi \Lambda(r)|\sin \theta|-2 \Lambda_{e}(r) \theta \sin \theta+r \cos \theta \int_{-r}^{r} \frac{\Lambda(u)}{u^{2}} d u
$$

apart from an error term $\eta r \log |2 \csc \theta|$, where $\eta \rightarrow 0$ uniformly in $0<|\theta|<\pi$ as $r \rightarrow \infty$. This improves theorems of Pfluger, Kahane and Rubel, Cartwright, and others, in that we do not assume existence of $\lim A(u) / u$, we do not assume that $F$ is entire or even, and the error term has a convergent integral with respect to $\theta$. Similar theorems for functions with negative poles and zeros, given later in the paper, generalize other familiar results. Here the error term involves $\log \left(2 \sec \frac{1}{2} \theta\right)$.

Another kind of result is briefly described as follows: Let $R=R(x)$ and $S=S(x)$ be positive functions such that the ratios $x / R, R / x, x / S, S / x$ are bounded as $|x| \rightarrow \infty$. Then for many purposes the function

$$
\log F(z)-z \int_{-|x|}^{|x|} \frac{\Lambda(u)}{u^{2}} d u
$$

can be replaced by the function

$$
\Lambda^{*}(z ; R, S)=\int_{x-R}^{x+S} \frac{\Lambda(u)}{z-u} d u .
$$

This remark is given content by detailed estimates of the error and of $\Lambda^{*}$. (For simplicity of statement the text takes $R=S$ but the form mentioned here is sometimes more con- 
venient, and is allowed by the proofs.) The development leads to a simple, systematic method of proving variety of growth theorems. Formulation of such a method is a principal goal of this paper, and our specific examples are intended only for illustration.

1. Some preliminary matters. We begin by giving a precise description of the class of functions being considered, and we also introduce some notation. With $\lambda_{n}$ and $\mu_{n}$ as described above, our objective is to estimate the magnitude of the expression

$$
F(z)=\prod_{-\infty}^{\infty} \frac{W\left(z, \lambda_{n}\right)}{W\left(z, \mu_{n}\right)}
$$

where $W$ denotes the Weierstrass primary factor,

$$
W(z, \lambda)=\left(1-\frac{z}{\lambda}\right) \exp \left[\frac{z}{\lambda}+\frac{1}{2}\left(\frac{z}{\lambda}\right)^{2}+\cdots+\frac{1}{p}\left(\frac{z}{\lambda}\right)^{p}\right] .
$$

The product is interpreted by taking all factors for which $\lambda_{n}$ and $\mu_{n}$ lie on the interval $(-R, R)$ and then letting $R \rightarrow \infty$. Thus, if $\Lambda=$ $\Lambda_{1}-\Lambda_{2}$, we have

$$
\log F(z)=\lim _{R \rightarrow \infty} \int_{-R}^{R} \log W(z, u) d \Lambda(u),
$$

when the limit exists. A suitable branch of the logarithm is easily determined in the upper half plane or in the lower half plane, and the relation between these branches is established by requiring that integrals of form

$$
\int_{-\infty}^{\infty} K(u, z) A(u) d u
$$

be always even or odd, according as $K$ is even or odd. To keep track of these matters, we never introduce angles larger than $\pi$. Thus

$$
z=x+i y=r e^{i \theta}, \text { where } r>0 \text { and }-\pi<\theta \leqq \pi .
$$

The letters $z, x, y, r, \theta$ are used in this sense, whereas the letters $C, D^{+}, D^{-}, D, \rho, \alpha, \beta, \gamma$ denote constants.

The decomposition of a function into even and odd parts is expressed by use of subscripts $e$ and $o$. Thus, for any function $\phi$ we define $\phi=\dot{\phi}_{e}+\phi_{o}$ where $\phi_{e}$ is even, $\phi_{o}$ is odd, and $\phi_{0}(0)=0$. We also set

$$
\Lambda=\Lambda_{1}-\Lambda_{2}, \quad A(u)=u^{-p} \Lambda(u), \quad \widetilde{A}(r)=\int_{-r}^{r} \frac{A(u)}{u} d u,
$$

where $p=0,1,2, \cdots$ and $-\infty<u<\infty$, but $0<r<\infty$. The letter 
$F$ is reserved for functions which satisfy the hypothesis, and hence the conclusion, of the following elementary lemma. (The designation as "Theorem 1" does not describe the status of the assertion, but serves to facilitate cross reference. A similar convention is used for lemmas and corollaries throughout this paper.)

Theorem 1 (lemma). Let $A_{e}(u)=o(u), A_{o}(u)=o\left(u^{2}\right)$, and suppose that

$$
\int_{0}^{\infty} u^{-2} A_{e}(u) d u \text { and } \int_{0}^{\infty} u^{-3} A_{o}(u) d u
$$

exist. Then the limit defining $F(z)$ exists for $z \neq \mu_{n}$, and if also $z \neq \lambda_{n}$

$$
\log F(z)=z^{p} \int_{0}^{\infty}\left[u A_{e}(u)+z A_{o}(u)\right] \frac{2 z}{u\left(z^{2}-u^{2}\right)} d u .
$$

For proof, integrate by parts in (3). The integrated terms have the form

$$
\left(\frac{2}{p+1} \frac{A_{e}(R)}{R} z^{p+1}+\cdots\right)+\left(\frac{2}{p+2} \frac{A_{o}(R)}{R^{2}} z^{p+2}+\cdots\right)
$$

and disappear as $R \rightarrow \infty$. We have, therefore,

$$
\log F(z)=z^{p} \lim _{R \rightarrow \infty} \int_{-R}^{R} A(u) \frac{z}{u(z-u)} d u
$$

provided the latter limit exists. Replacing $u$ by $-u$ gives another form for the integral (5), and still a third form is obtained by taking half the sum of these two. In terms of $A_{e}$ and $A_{o}$ the third form is

$$
\begin{aligned}
& \frac{z}{2} \int_{-R}^{R} \frac{A_{e}(u)}{u}\left(\frac{1}{z-u}-\frac{1}{z+u}\right) d u \\
& \quad+\frac{z}{2} \int_{-R}^{R} \frac{A_{o}(u)}{u}\left(\frac{1}{z-u}+\frac{1}{z-u}\right) d u .
\end{aligned}
$$

Inasmuch as $\left(z^{2}-u^{2}\right)^{-1}=u^{-2}+O\left(u^{-4}\right)$ the integrals in (6) converge as $R \rightarrow \infty$, and this gives Theorem 1 .

Formulas similar to (5) are well known in the theory of entire functions. But since $\Lambda=\Lambda_{1}-\Lambda_{2}$, and since the factor $u^{p}$ has been divided out in the definition of $A$, the present assumptions are weaker than the customary ones. For example when $p=1$ the even part of $\Lambda$ is required to satisfy only

$$
\Lambda_{s}(u)=o\left(u^{3}\right), \quad \int_{0}^{\infty} u^{-4} \Lambda_{e}(u) d u \text { exists . }
$$


Regardless of the value of $p$, both $\left\{\lambda_{n}\right\}$ and $\left\{\mu_{n}\right\}$ can belong to products of infinite genus without invalidating the conclusion.

2. An asymptotic formula. By analogy with familiar properties of entire functions one can surmise that regular behavior of $F$ follows from existence of the two limits

$$
\lim _{u \rightarrow-\infty} A(u)=D^{-}, \quad \lim _{u \rightarrow \infty} A(u)=D^{+} .
$$

We shall see that this is the case and in fact, that the weaker condition

$$
\lim _{r \rightarrow \infty}[A(r u)-A(r / u)]=0, \quad(u \neq 0), \quad A(u)=O(1)
$$

introduced by the author [11] is sufficient. Evidently (7) implies (8) but the converse is false even if $\lambda_{n}$ and $\mu_{n}$ are integers.

TheOREm 2. Suppose $A(u)$ is bounded and $A(u r)-A(r / u) \rightarrow 0$ as $r \rightarrow \infty$, for each fixed $u \neq 0$. Then

$$
\begin{aligned}
& r^{-p} \log \left|F\left(r e^{i \theta}\right)\right| \\
& \quad=\left[\pi A(r) \operatorname{sgn} \theta-2 A_{o}(r) \theta\right] \sin p \theta+\widetilde{A}(r) \cos p \theta+\eta \log |2 \csc \theta|
\end{aligned}
$$

where $\eta \rightarrow 0$ uniformly in $0<|\theta|<\pi$ as $r \rightarrow \infty$.

We have to write $\log |2 \csc \theta|$ rather than $\log |\csc \theta|$ because of the accident that the latter function vanishes at $\theta=\pi / 2$. The assertion of uniformity is relevant only near 0 and $\pi$.

Upon taking $r=u s$ we find that the first condition (8) is equivalent to the two conditions

(9) $\lim _{r \rightarrow \infty}\left[A_{e}(r t)-A_{e}(r)\right]=0, \lim _{r \rightarrow \infty}\left[A_{o}(r t)-A_{o}(r)\right]=0, \quad t>0$.

By Theorem $1 \log |F(z)|=\operatorname{Re}\left[z^{p}\left(I_{e}+I_{o}\right)\right]$ where

$$
I_{e}=\int_{-\infty}^{\infty} \frac{A_{e}(u)}{z^{2}-u^{2}} z d u, \quad I_{o}=\int_{-\infty}^{\infty} \frac{A_{o}(u)}{u\left(z^{2}-u^{2}\right)} z^{2} d u
$$

An easy contour integration gives

$$
\int_{-\infty}^{\infty} \frac{z}{z^{2}-u^{2}} d u=-\pi i \operatorname{sgn} y
$$

and hence, $I_{e}$ can be estimated as in [5]. That is, multiply (11) by $\mathrm{A}_{e}(r)$, subtract from (10), and let $u=t r$. The result is

$$
I_{e}=2 \int_{0}^{\infty} \frac{A_{e}(r t)-A_{e}(r)}{z^{2}-r^{2} t^{2}} z r d t-\pi i A_{e}(r) \operatorname{sgn} y .
$$

The integral is now analyzed by breaking the range at $\delta$ and $1 / \delta$, 
where $\delta$ is a small positive constant. In this decomposition the first and third integrals are uniformly $O(\delta)$ by inspection, since $|A(u)|$ is bounded. The second does not exceed

$$
\eta_{1}(r) \int_{\delta}^{1 / \delta} \frac{r^{2}}{\left|z^{2}-r^{2} t^{2}\right|} d t
$$

where $\eta_{1}$ is a bound for $\left|A_{e}(r t)-A_{e}(r)\right|$ on the interval $\delta \leqq t \leqq 1 / \delta$. Since the limits (9) are known to be automatically uniform [6] on any fixed interval $(\delta, 1 / \delta)$ we have $\eta_{1}(r) \rightarrow 0$ as $r \rightarrow \infty$.

The integral multiplying $\eta_{1}(r)$ in (12) does not exceed the value it would have for $\delta=0+$, namely,

$$
\int_{0}^{\infty} \frac{r}{\left|z^{2}-u^{2}\right|} d u=\int_{0}^{\infty} \frac{d t}{\left(1-2 t^{2} \cos 2 \theta+t^{4}\right)^{1 / 2}}=K(\cos \theta)
$$

where $K$ denotes the complete elliptic integral of the first kind. By an elementary calculation

$$
K(\cos \theta) \sim \log |\csc \theta| \quad(\theta \rightarrow 0 \text { or } \pi)
$$

and hence, the desired estimate is obtained if we choose $\delta$ first, then $r$.

Estimation of $I_{o}$ depends on the observation that

$$
I_{o}=\widetilde{A}(r)+2 \int_{0}^{1} \frac{A_{o}(t r)}{z^{2}-t^{2} r^{2}} r^{2} t d t+2 \int_{1}^{\infty} \frac{A_{o}(t r)}{t\left(z^{2}-t^{2} r^{2}\right)} z^{2} d t .
$$

In order to replace $A_{o}(t r)$ by $A_{o}(t r)-A_{o}(r)$, consider the sum

$$
j(\omega)=\int_{0}^{1} \frac{t d t}{\omega-t^{2}}+\int_{1}^{\infty} \frac{\omega d t}{t\left(\omega-t^{2}\right)}, \quad \omega=e^{2 i \theta},
$$

which arises when $A_{o}(t r)$ is replaced by 1 . Setting $t=1 / s$ in the second integral produces an integral much like the first, so that

$$
j(\omega)=i\left(\theta-\frac{\pi}{2} \operatorname{sgn} \theta\right)
$$

by a short calculation. We now write

$$
\begin{aligned}
I_{o}= & \widetilde{A}(r)+2 j(\omega) A_{o}(r)+2 \int_{0}^{1} \frac{A_{o}(t r)-A_{o}(r)}{z^{2}-t^{2} r^{2}} r^{2} t d t \\
& +2 \int_{1}^{\infty} \frac{A_{o}(t r)-\mathrm{A}_{o}(r)}{t\left(z^{2}-t^{2} r^{2}\right)} z^{2} d t .
\end{aligned}
$$

The range of integration is divided at $\delta$ and $1 / \delta$ in the first and second integrals, respectively. Of the resulting four integrals the first is $O(\delta)$ and the last is $O\left(\delta^{2}\right)$, by inspection. The two others together are dominated by 


$$
\int_{\delta}^{1 / \delta} \frac{A_{o}(t r)-A_{o}(r)}{z^{2}-t^{2} r^{2}} r^{2} d t
$$

Since this has the same form as the expression leading to (12), the desired estimate is obtained by choosing $\delta$ first, then $r$.

The result of this analysis is

$$
\log F(z)=-\pi i A_{e}(r) \operatorname{sgn} \theta z^{p}+\widetilde{A}(r) z^{p}+i(2 \theta-\pi \operatorname{sgn} \theta) A_{o}(r) z^{p}
$$

apart from a term $\eta z^{p} \log |2 \csc \theta|$, where $\eta$ is uniformly small as $r \rightarrow \infty$. Upon taking real parts and dividing by $r^{p}$ we get Theorem 2 .

3. Discussion and illustrations. If the main assumption on $A$ is replaced by

$$
\begin{aligned}
& \lim _{r \rightarrow \infty} \int_{\delta}^{1 / \delta}\left|A_{e}(r t)-A_{e}(r)\right| d t=0, \\
& \lim _{r \rightarrow \infty} \int_{\delta}^{1 / \delta}\left|\mathrm{A}_{o}(r t)-A_{o}(r)\right| d t=0
\end{aligned}
$$

for each $\delta>0$, then the same conclusion holds in Theorem 2, except that the term $\log |2 \csc \theta|$ must be replaced by the larger term $|\csc \theta|$. (The condition that $A$ be bounded can also be weakened, as is evident.) To prove the sufficiency of (13) we need only observe that

$$
\left|\frac{z r \sin \theta}{z^{2}-r^{2} t^{2}}\right|=\frac{1}{2}\left|\frac{e^{2 i \theta}-1}{e^{2 i \theta}-t^{2}}\right| \leqq 1 \text {. }
$$

The advantage of the term $\log |2 \csc \theta|$ is that it has a convergent integral with respect to $\theta$. More generally, let $F$ be a function satisfying the conclusion of Theorem 2 , with the error term $\eta \log |2 \csc \theta|$ replaced by any function $\eta(r, \theta)$ such that

$$
\lim _{r \rightarrow \infty} \int_{-\pi}^{\pi} \eta(r, \theta) d \theta=0
$$

By Jensen's theorem

$$
\widetilde{\Lambda}(r)=\frac{1}{2 \pi} \int_{-\pi}^{\pi} \log \left|F\left(r e^{i \theta}\right)\right| d \theta,
$$

and hence substitution of the expression of Theorem 2, with such an error term, gives

$$
\frac{p}{r^{p}} \int_{-r}^{r} u^{p-1} A(u) d u=A_{e}(r)\left[1-(-)^{p}\right]+A_{o}(r)\left[1+(-)^{p}\right]+o(1)
$$

for $p \geqq 1$. From this it follows that 


$$
\int_{0}^{1}\left[A_{o}(r t)-A_{o}(r)\right] d t \rightarrow 0 \quad \text { or } \quad \int_{0}^{1}\left[A_{e}(r t)-A_{e}(r)\right] d t \rightarrow 0
$$

as $r \rightarrow \infty$, for $p$ even or $p$ odd, respectively. In short, conditions of the type (13) are close to being necessary as well as sufficient.

We now consider some special cases. When $F$ is even then $F(\lambda)=0$ if and only if $F(-\lambda)=0$, and the same applies to $1 / F$. Hence $\Lambda$ is odd, and we conclude

$$
A(u)=A_{e}(u) \quad(p \text { odd }), \quad A(u)=A_{o}(u) \quad(p \text { even }) .
$$

The corresponding simplified form of Theorem 2 can be read off by inspection. In particular, the case $p=1, F$ even, gives

$$
\log \left|F\left(r e^{i \theta}\right)\right|=\pi \Lambda(r)|\sin \theta|+o(r) \log |2 \csc \theta| .
$$

If we specialize further by making $\Lambda_{2}=0$ (so that $F$ is entire) then this is a result of Kahane and Rubel [5], supplemented by the additional information concerning uniformity of the error.

To introduce the next example define

$$
h(\theta)=\lim _{r \rightarrow \infty} \sup r^{-p} \log \left|F\left(r e^{i \theta}\right)\right|
$$

as is customary, and also

$$
\alpha=\lim _{r \rightarrow \infty} \sup \left[|A(r)|+\left|A_{o}(r)\right|\right], \quad \widetilde{\alpha}=\lim _{r \rightarrow \infty} \sup \widetilde{A}(r) .
$$

For $0<|p \theta|<\pi / 2$ Theorem 2 gives

$$
\tilde{\alpha} \cos p \theta-\pi \alpha|\sin p \theta| \leqq h(\theta) \leqq \widetilde{\alpha} \cos p \theta+\pi \alpha|\sin p \theta| .
$$

Because of the poles of $F(z)$ we cannot get any estimate for $\theta=0$. But if $\Lambda_{2}=0$ then $F$ is an entire function of order $p$, under the hypothesis of Theorem 2, and continuity of the Phragmén-Lindelöf function gives:

THEOREM 3 (corollary). Let $F$ be an entire function satisfying the hypothesis of Theorem 2. Then

$$
\lim _{x \rightarrow \infty} \sup \frac{\log |F(x)|}{x^{p}}=\lim _{x \rightarrow \infty} \sup \int_{-x}^{x} \frac{\Lambda(u)}{u^{p+1}} d u
$$

if the latter limit is positive, and otherwise the left side is $\leqq 0$.

There is a similar theorem for $x \rightarrow-\infty$ which distinguishes the cases: $p$ even, $p$ odd. If $F$ is even and $p$ is odd, the integral in Theorem 3 vanishes identically, and the conclusion is $\log |F(x)| \leqq o\left(x^{p}\right)$. The special case $p=1$ is in [11], though the proof here follows [5]. To show the connection of our results with the classical theory 
of entire functions, introduce the sum

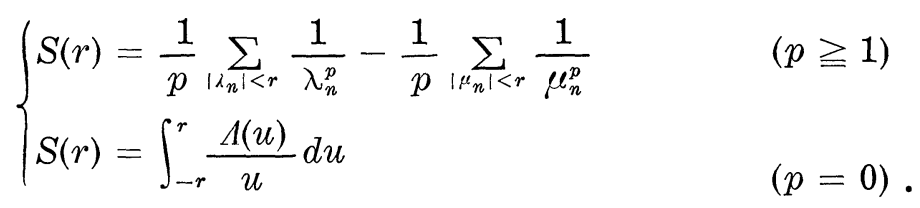

By partial integration

$$
S(r)=2 p^{-1} A_{0}(r)+\widetilde{A}_{0}(r), \quad p \geqq 1,
$$

giving an alternative form of Theorem 2. As a special case, if $\lim A(u)=D$ for $|u| \rightarrow \infty$, then

$$
r^{-p} \log \left|F\left(r e^{i \theta}\right)\right|-\pi D \operatorname{sgn} \theta \sin p \theta-S(r) \cos p \theta=\eta \log |2 \csc \theta|
$$

where $\eta \rightarrow 0$ uniformly in $0<|\theta|<\pi$ as $r \rightarrow \infty$. For proof note that (8) holds and $\lim A_{o}(r)=0, \lim A_{e}(r)=D$. Thus the result follows by substitution in Theorem 2 . The choice $\Lambda_{2}=0, p=1$ gives a theorem of Pfluger [2], supplemented by the uniformity of the error term. If we specialize further by taking $D=0$ the result is a theorem of Cartwright [2], also supplemented.

4. Evaluation of the error. Besides the function $\widetilde{A}$ introduced in $\S 1$ we require

$$
A^{*}(z, R)=\int_{x-R}^{x+R} \frac{A(u)}{z-u} d u, \quad R>0 .
$$

The result of applying the operator * to any function is here called an incomplete Hilbert transform of that function, because it becomes a Hilbert transform when $R=\infty$. Both $\widetilde{A}$ and $A^{*}$ are, as a rule, principal terms in our estimates. The former is important if the growth of $A$ is moderately fast, while the latter is important near the real axis.

By contrast, the expressions

$$
A^{m}(r)=\int_{0}^{r}\left(\frac{u}{r}\right)^{m} \frac{|A(u)|}{u} d u+\int_{r}^{\infty}\left(\frac{r}{u}\right)^{m} \frac{|A(u)|}{u} d u, \quad m>0
$$

are in the nature of error terms. We take the view that the $\sim$ in $\widetilde{A}$, the ${ }^{*}$ in $A^{*}$, and the superscript in $A^{m}$ denote operations to be performed on any function to which they are applied. Thus, $\mathrm{A}_{o}^{2}$ is obtained from (19) with $m=2$ and with $A_{o}$ replacing $A$.

THEOREM 4. For $0<|\theta|<\pi$ we have

$$
\left|z^{-p} \log F(z)-\widetilde{A}(r)\right| \leqq 2|\csc \theta|\left[A_{e}^{1}(r)+A_{o}^{2}(r)\right]
$$


and for $2 x^{2} \geqq r^{2},|x| \geqq R$ we have

$$
\left|z^{-p} \log F(z)-\widetilde{A}(|x|)-A^{*}(z, R)\right| \leqq 4 \frac{|x|}{R}\left[A_{e}^{1}(|x|)+A_{o}^{2}(|x|)\right] .
$$

To prove the first assertion set

$$
k_{1}=\frac{2 z^{2}}{z^{2}-u^{2}}, \quad k_{2}=\frac{2 u^{2}}{z^{2}-u^{2}} .
$$

The integral multiplying $z^{p}$ in Theorem 1 can be written

$$
\widetilde{A}_{o}(r)+\int_{0}^{r}\left(\frac{A_{e}}{z}+\frac{A_{o} u}{z^{2}}\right) k_{1} d u+\int_{r}^{\infty}\left(\frac{z A_{e}}{u^{2}}+\frac{z^{2} A_{o}}{u^{3}}\right) k_{2} d u .
$$

Since $\left|k_{i} \sin \theta\right| \leqq 2$, this gives the desired estimate.

To get the second estimate define

$$
k_{3}=\frac{z}{z+u}, \quad k_{4}=\frac{2 z+u}{u(z+u)} .
$$

When $x>0$ the conclusion of Theorem 1 gives

$$
z^{-p} \log F(z)-\widetilde{A}(x)-A^{*}(z, R)
$$

$$
\begin{aligned}
= & \int_{0}^{x-R}\left(\frac{A_{e}}{z}+\frac{u A_{o}}{z^{2}}\right) k_{1} d u+\int_{x-R}^{x} \frac{A_{e}-A_{o}}{z} k_{3} d u \\
& +\int_{x+R}^{\infty}\left(\frac{z A_{e}}{u^{2}}+\frac{z^{2} A_{o}}{u^{3}}\right) k_{2} d u+\int_{x}^{x+R} \frac{A_{e}}{z} k_{3} d u+\int_{x}^{x+R} A_{o} k_{4} d u .
\end{aligned}
$$

If $2 R \leqq x$ and $0 \leqq u \leqq x-R$, it is possible to show that

$$
\left|k_{1}\right| \leqq \frac{2 x^{2}}{x^{2}-u^{2}} \leqq \frac{4 x}{3 R},
$$

and evidently $\left|k_{3}\right| \leqq 1$ for $u>0$. Hence (20) admits an estimate somewhat sharper than

$$
\frac{4 x}{3 R} \int_{0}^{x}\left(\frac{\left|A_{e}\right|}{r}+\frac{u\left|A_{o}\right|}{r x}\right) d u .
$$

Similarly, if $u \geqq x+R$ and $2 R \leqq x$, then

$$
\left|k_{2}\right| \leqq \frac{2 u^{2}}{u^{2}-x^{2}} \leqq \frac{9 x}{5 R},
$$

and for $x \leqq u \leqq(3 / 2) x$ it is possible to show that $\left|k_{4}\right| \leqq 18 r^{2} /\left(5 u^{3}\right)$. For $2 R \leqq x$ the expression (21) is therefore dominated by

$$
\frac{9 x}{5 R} \int_{x}^{\infty}\left(\frac{\left|A_{e}\right| r}{u^{2}}+\frac{\left|A_{o}\right| r^{2}}{u^{3}}\right) d u \text {. }
$$


Addition of (22) and (23) gives an estimate which is sharper than that of the theorem when $r^{2} \leqq 2 x^{2}$.

So far we have assumed $x>0$. If $\phi(z)=z^{-p} \log F(z)$ then $\phi(-z)$ is obtained by writing $-A_{e}(u)$ instead of $A_{e}(u)$ in the formula for $\phi(z)$ (Theorem 1). Upon noting the effect of this substitution on $\widetilde{A}_{o}$, on $A^{*}$, and on the error terms, we obtain Theorem 4 .

The restriction $2 x^{2} \leqq r^{2}$ means that $|\sin \theta| \leqq(1 / 2) \sqrt{2}$, in other words, $z$ lies in a 90-degree sector centered about the positive or negative real axis. Now, a restriction of the type $|\sin \theta| \leqq \delta$ is entirely harmless, because for other values of $z$ the first estimate can be used. It is therefore relevant to note that, if $\sin \theta=O(\sqrt{\delta})$ and $R=|x| O(\delta)$, then the coefficient " 4 " in the second error term can be replaced by $1+O(\delta)$ as $\delta \rightarrow 0+$. This is true because the stated conditions give

$$
R\left|k_{1}\right| \leqq|x|, \quad R\left|k_{2}\right| \leqq|x|, \quad 2\left|k_{3}\right| \leqq 1, \quad 2\left|x k_{4}\right| \leqq 3
$$

apart from a factor $1+O(\delta)$, and $r=|x|$ apart from the same factor.

The second assertion of Theorem 4 remains valid if the term $4 R^{-1}|x| A_{e}^{1}(|x|)$ is replaced by

$$
4 \frac{|x|^{2}}{R} \int_{|x|}^{\infty} \frac{\left|A_{e}(u)\right|}{u^{2}} d u+\frac{1}{r} \int_{0}^{|x|}\left|A_{e}(u)\right| d u+\frac{|x|}{r} \int_{0}^{|x|-R} \frac{\left|A_{e}(u)\right|}{|x|-u} d u .
$$

Proof of this requires a somewhat different estimate for the part of the error due to $A_{e}$ on $(0, x), x>0$. By (20) this part of the error does not exceed

$$
\frac{1}{r} \int_{0}^{x-R} \frac{2 x^{2}}{x^{2}-u^{2}}\left|A_{e}(u)\right| d u+\frac{1}{r} \int_{x-R}^{x}\left|A_{e}(u)\right| d u .
$$

The conclusion (24) now follows from the inequality

$$
\frac{2 x^{2}}{x^{2}-u^{2}} \leqq 1+\frac{x}{x-u} \quad(x>0, u>0) \text {. }
$$

5. The classes $B_{\rho}$ and $M_{\rho}$. As noted in $\S 1, r \geqq 0$. If $\phi=\phi(r)$ is a function of $r$ the statement " $\phi \in B_{\rho}$ " means that $\phi$ is integrable and

$$
\int_{1}^{\infty} \frac{|\phi(r)|}{r^{\rho+1}} d r<\infty, \quad \rho=\text { const } .
$$

On the other hand functions of $u$, such as $A(u)$, are defined for $-\infty<u<\infty$. The statement $A \in B_{\rho}$ then means that (25) holds for $\phi(r)=A(r)$ and for $\phi(r)=A(-r)$. Thus both the even and odd parts, $A_{e}$ and $A_{o}$, have to satisfy (25).

If $|\phi(r)|$ admits an increasing majorant $\phi^{+}(r) \in B_{\mathrm{\rho}}$ then we say $\phi \in B_{\rho}^{+}$. In agreement with the above convention a function such as 
$A(u)$ belongs to $B_{\rho}^{+}$if $|A(r)| \leqq \phi(r)$ and $|A(-r)| \leqq \phi(r)$, where $\phi$ is an increasing function satisfying (25).

If $\phi=\phi(r)$ is a function of $r$ the statement " $\phi \in M_{\rho}$ " means that $\rho \geqq 0$, and that $\phi(r) r^{\rho}$ is increasing but $\phi(r) r^{-\rho}$ is decreasing. For functions of $u$ rather than $r$ the same convention is used as in the case $B$. Thus, the even and odd parts must belong to $M_{\rho}$, taking $u=r \geqq 0$.

Theorem 5 (lemma). Let $\phi \geqq 0$ be integrable, let $\phi=0$ near 0 , and for $m>0$ define

$$
\tilde{\phi}(r)=\int_{0}^{r} \frac{\phi(u)}{u} d u, \quad \phi^{m}(r)=\int_{0}^{r}\left(\frac{u}{r}\right)^{m} \frac{\phi(u)}{u} d u+\int_{r}^{\infty}\left(\frac{r}{u}\right)^{m} \frac{\phi(u)}{u} d u .
$$

\section{Then:}

(i) $\phi \in M_{\rho}$ implies $(m-\rho) \phi^{m} \leqq 2 \phi$.

(ii) $\phi \in B_{\rho}$ implies $\tilde{\phi} \in B_{\rho}^{+}$for $\rho>0$ and $\phi^{m} \in B_{\rho}$ for $|\rho|<m$.

(iii) If $r^{\alpha} \phi(r)$ is increasing for some $\alpha$, then $\phi \in B_{\rho} \Rightarrow \phi \in B_{\rho}^{+}$for $\rho>0$.

(iv) $\phi \in B_{\rho}$ implies $r^{\alpha} \phi^{m}(r) \in B_{\rho+\alpha}^{+}$for $\rho+\alpha>0,|\rho|<m$.

(v) $\phi(r) \log r \in B_{\rho} \Rightarrow \phi^{m} \in B_{\rho}^{+}$for $\rho=m$.

The statement (i) is trivial and (ii) follows by a change in order of integration, as is well known. To get (iii) consider $\tilde{\phi}(2 r)-\widetilde{\phi}(r)$. Under the given hypothesis it is found that $\phi(r) \leqq \gamma \tilde{\phi}(2 r)$ for some constant $\gamma$. But $\tilde{\phi}(r) \in B_{\rho}^{+}$by (ii), and hence $\tilde{\phi}(2 r)$ has the same property. The statement (iv) follows from

$$
\phi \in B_{\rho} \Longrightarrow \phi^{m} \in B_{\rho} \Longrightarrow r^{\alpha} \dot{\phi}^{m} \in B_{\rho+\alpha} \Longrightarrow r^{\alpha} \dot{\phi}^{m} \in B_{\rho+\alpha}^{+} .
$$

In the last step we used (iii) together with the observation that $r^{m} \phi^{m}(r)$ is nondecreasing for $m>0$. Proof of $(\mathrm{v})$ is similar.

In (iii) the purpose of assuming $r^{\alpha} \phi(r)$ increasing is to ensure an inequality of form $\phi(r) \leqq \gamma \widetilde{\phi}(\beta r)$ for large $r$. The same end would be accomplished by the weaker condition

$$
\int_{\alpha}^{\beta}[\phi(t r)-\delta \phi(r)] \frac{d t}{t} \geqq 0, \quad r \geqq r_{o},
$$

where $\alpha, \beta$ and $\delta$ are constant, with $\beta>\alpha \geqq 0, \delta>0$. The class $M_{\rho}$ can be generalized similarly, without invalidating our conclusions.

Conditions of the type (26a) are useful for getting an estimate on $\Lambda(u)$ from Jensens's theorem (15), since the latter gives $\widetilde{\Lambda}(r)$. Thus if $\phi(u) \equiv \Lambda(u)$ satisfies (26a) together with

$$
\phi_{o}(|u|) \geqq \varepsilon|\phi(\varepsilon u)|, \quad|u|>u_{o},
$$


for some positive constant $\varepsilon$, then

$$
\widetilde{\Lambda}(r) \leqq O[H(r)] \Longrightarrow|\Lambda(r)| \leqq O[H(r)]
$$

for every function $H \in M_{\rho}$, and furthermore,

$$
\widetilde{\Lambda}(r) \in B_{\rho} \Longrightarrow \Lambda(u) \in B_{\rho}
$$

for every $\rho$. These conditions are fulfilled for many meromorphic functions [and for all entire functions, since in that case $\Lambda_{o}(r)$ is increasing and $\left.|\Lambda(u)| \leqq 2 \Lambda_{o}(|u|)\right]$.

6. The incomplete Hilbert transform. We now estimate the incomplete transform, $A^{*}$. If $D \geqq 0$ is constant, denote by $K(D)$ the class of functions $A$ such that $A(u)+D u$ is nondecreasing; for example

$$
\Lambda_{1}(u)-D u \in K(D) \text {. }
$$

We say " $x$ and $\lambda_{n}$ are separated by $c$ " if $c$ is a positive constant such that the conditions

$$
\lambda_{n+1}-\lambda_{n} \geqq c, \quad\left|x-\lambda_{n}\right| \geqq c
$$

hold for all $n$.

Theorem 6 (lemma). Let $\phi$ be integrable and for $|u-x| \leqq R$ define

$$
M=\sup |\phi(u)|, \quad J=\int_{x-R}^{x+R}|\phi(u)| d u, \quad \phi^{*}=\int_{x-R}^{x+R} \frac{\phi(u)}{z-u} d u .
$$

\section{Then:}

(i) $\left|\operatorname{Im} \phi^{*}\right| \leqq \pi M,\left|\operatorname{Re} \phi^{*}\right| \leqq 2 M \log |\csc \theta|,\left|\phi^{*}\right| \leqq 2 M \log |\csc \theta|$ for all $R$, for $R \leqq|x|$, and for $\max (4 R, 2|y|) \leqq|x|$, respectively.

(ii) The condition $\phi \in K(D)$ implies $\operatorname{Re} \phi^{*} \leqq 2 \min (D R, \sqrt{D J})$.

(iii) If $\lambda_{n}$ and $x$ are separated by $c$, then $\operatorname{Re} \Lambda^{*} \geqq-2 R c^{-1}$.

The statement (i) follows from the observation that

$$
\begin{aligned}
& \int_{x-R}^{x+R}\left|\frac{1}{z-u}\right| d u=2 \log \cot \frac{1}{2} \psi, \\
& \int_{x-R}^{x+R}\left|\operatorname{Re} \frac{1}{z-u}\right| d u=2 \log \csc \psi
\end{aligned}
$$

where $\psi$ is the first-quadrant angle defined by

$$
\sin \psi=\frac{|y|}{\left(R^{2}+y^{2}\right)^{1 / 2}} .
$$


To establish (ii) define

$$
I(\phi) \equiv \operatorname{Re} \phi^{*}(z, R)=\int_{x-R}^{x+R} \frac{x-u}{(x-u)^{2}+y^{2}} \dot{\phi}(u) d u .
$$

If $\phi \in K(D)$ then $\phi(u)=D(x-u)+\psi(u)$ where $\psi$ is increasing. Therefore $I(\psi) \leqq 0$, and we obtain

$$
I(\phi)=I[D(x-u)]+I(\psi) \leqq I[D(x-u)] \leqq 2 D R .
$$

Proof of the inequality involving $J$ depends on the fact that for $y=0$ the kernel in (28) not only changes sign at $x$, but is monotone on each side of $x$. We carry out the proof by maximizing $I(\phi)$ subject to

$$
J(\phi) \equiv \int_{x-R}^{x+R}|\phi(u)| d u=J_{o},
$$

where $J_{o}$ is constant. We can suppose $\sqrt{D J_{0}} \leqq D R$, since otherwise the desired estimate follows from the estimate already obtained.

If $\phi(u)=0$ at $u=x$, the maximizing function must be positive for $u<x$ and negative for $u>x$. (Otherwise we could increase $I$ without changing $J$.) Thus, $(x-u) \phi(u) \geqq 0$, the value of $I$ increases as $|y|$ decreases, and hence we can assume $y=0$. Since $(x-u)^{-1}$ is monotone for $u<x$, the area at the left of $x$ should be pushed as close to $x$ as possible. Similarly the (negative) area at the right of $x$ should be pushed as close to $x$ as possible. The maximizing function for $y=0$ therefore follows the line $s=D(x-u)$ from $x-\alpha$ to $x+\beta$, say, and is zero elsewhere.

Because of the symmetry of $(x-u)^{-1}$, if $\alpha>\beta$ we could do better by transferring some of the area from the left to the right, and similarly if $\alpha>\beta$. Thus $\alpha=\beta$, and the maximum value is $2 D \alpha$ as in the previous discussion. Since $D \alpha^{2} \leqq J_{0}$, this gives the final result.

We must still justify our initial assumption that $\phi=0$ at the central point, $x$. The following elegant discussion, simpler than the author's, is due to Ernst Straus. Let $\phi=\phi_{1}+\phi_{2}$, where $\phi_{1}$ is even about the point $x$ and $\phi_{2}$ is odd, with $\phi_{2}(x)=0$. Then

$$
\dot{\phi} \in K(D) \Rightarrow \phi_{2} \in K(D), \quad I(\dot{\phi})=I\left(\phi_{2}\right), \quad J(\dot{\phi}) \geqq J\left(\phi_{2}\right) .
$$

Indeed, the first relation can be seen from the fact that $\phi \in K(D)$ is equivalent to $\phi^{\prime} \geqq-D$ when $\phi$ is differentiable. The second is obvious, and the third follows from

$$
2 \int_{0}^{R}\left|\phi_{2}\right| d u \leqq \int_{0}^{R}\left(\left|\phi_{1}-\phi_{2}\right|+\left|\phi_{1}+\phi_{2}\right|\right) d u .
$$

Therefore $\phi$ can be replaced by $\phi_{2}$, giving $\phi(0)=\phi_{2}(0)=0$. 
The somewhat informal language of the foregoing discussion is best suited to a restricted class of functions $\phi$, such as the class of piecewise continuous functions. (The $A$ in Theorem 1 is in fact piecewise continuous.) But since smooth functions are dense in the Lebesgue class $L$, we have actually established (ii) in full generality.

Under the hypothesis (iii) the condition $\Lambda(u)=0$ at $u=x$ implies that the graph of $s=\Lambda(u)$ lies above the line $s=c^{-1}(u-x)$ for $u<x$ and below the line for $u>x$. The foregoing method thus gives the conclusion.

7. Comparison. No use has been made of the fact that $A_{2}$ is an integer, and hence it is permissible to take $\Lambda_{2}(u)=\mathrm{D} u,|u|^{\rho} \operatorname{sgn} u$, and so on. The resulting estimates give information about the entire function associated with $\Lambda_{1}$. Thus many classical results on entire functions that seem unrelated to ours are in fact corollaries.

The same extension can be made without losing the applicability to meromorphic functions. Namely, if $A(u) \sim B(u)$ as $|u| \rightarrow \infty$, we compare $F(z)$ with the function $z^{p} G(z)$, where

$$
G(z)=\lim _{R \rightarrow \infty} \int_{-R}^{R} B(u) \frac{z}{u(z-u)} d u .
$$

It is supposed that $\widetilde{B}(r)$ exists as a Cauchy principal value, and the integral (31) therefore exists also, near 0 , as a Cauchy principal value. A suitable relation between the branches for $y>0$ and $y<0$ is found by the transformations leading to Theorem 1 . Since the integral operators in $\widetilde{A}, A^{*}$ and in Theorem 1 are linear, Theorem 6 remains valid if $z^{-p} \log F(z)$ is replaced by

$$
z^{-p} \log F(z)-G(z)
$$

and $A$ is replaced by $E=A-B$. For proof, assume that the integrals $E_{e}^{1}$ and $E_{o}^{2}$ converge; otherwise there is nothing to prove. Since $B=A-E$ the corresponding integrals with $B$ also converge (though perhaps not absolutely) and we can transform the integral for $G$ as in Theorem 1. This establishes the assertion.

For example, if $0<\rho<1$, our interpretation leads to

$$
\left\{\begin{array}{l}
\left.\int_{-\infty}^{\infty}|u|^{\rho} \frac{z}{u(z-u)} d u=\pi \csc \pi \rho \mid(-z)^{\rho}-z^{\rho}\right] \\
\int_{-\infty}^{\infty}|u|^{\rho} \operatorname{sgn} u \frac{z}{u(z-u)} d u=\pi \csc \pi \rho\left[(-z)^{\rho}+z^{\rho}\right]
\end{array}\right.
$$

where $0<|\theta|<\pi$. Here $z^{\rho}$ is real for $z>0$ and regular for $|z|>0$, $|\theta|<\pi$. Subtraction produces the familiar formula 


$$
\int_{0}^{\infty} u^{\rho} \frac{z}{u(z+u)} d u=\pi z^{\rho} \csc \pi \rho, \quad|\theta|<\pi .
$$

As another illustration, let $D_{o}(u)=D \operatorname{sgn} u$ for $|u| \geqq 1$, and $D_{o}(u)=0$ otherwise. Then

$$
\begin{aligned}
\int_{-\infty}^{\infty} D_{o}(u) \frac{z}{u(z-u)} d u & =D \log \left(1-z^{2}\right) \\
& =i D(2 \theta-\pi \operatorname{sgn} \theta)+2 D \log r+O\left(r^{-2}\right) .
\end{aligned}
$$

For $B(u)=D$ we have $G(z)=-\pi i D \operatorname{sgn} \theta$ by (11), and combining this with the above result for $D_{o}$ gives

$$
\begin{aligned}
B(u) & =D(u) \Rightarrow G(z) \\
& =i \theta\left(D^{+}-D^{-}\right)-D^{+} \pi i \operatorname{sgn} \theta+\widetilde{D}(r)+O\left(r^{-2}\right),
\end{aligned}
$$

where $D(u)$ is defined to be

$$
D^{-}, \quad \frac{1}{2}\left(D^{+}+D^{-}\right), \quad D^{+}
$$

for the range $u<-1,-1 \leqq u \leqq 1$, and $1<u$, respectively.

These equations give a suitable value for $G$, and the relation between $F$ and $G$ is then determined by Theorem 4. Analysis of the term $E^{*}$ is facilitated by the fact that

$$
P(z)[A(u)]^{*}-[P(u) A(u)]^{*}=\int_{x-R}^{x+R} \frac{P(z)-P(u)}{z-u} A(u) d u
$$

for any function $P(z)$. If $P$ satisfies a Lipschitz condition-and in particular if $P$ is analytic-the foregoing expression often admits a bound of the same order as our error terms. Thus, $[P(u) A(u)]^{*}$ can be replaced by the simpler expression $P(z)[A(u)]^{*}$. As an illustration,

$$
\left|z^{p} A^{*}(z, R)-A^{*}(z, R)\right| \leqq p(r+R)^{p-1} \int_{x-R}^{x+R}|A(u)| d u .
$$

This leads to an alternative form of Theorem 4, involving $A$ rather than $A$.

8. A theorem of Lindelö. When used in the manner described, Theorems 4-6 give a variety of specific results with the greatest ease.

THEOREM 7 (corollary). Let $S(r)$ be the sum (16) and let $A(u)$ $=O[H(|u|)]$ where $H \in M_{\rho},|\rho|<1$. Then the following statements are equivalent:

(i ) $r^{-p} \log \left|F\left(r e^{i \theta}\right)\right|=O[H(r)]$ for some $\theta$ with $\sin \theta \cos p \theta \neq 0$.

(ii) $r^{-p} \log \left|F\left(r e^{i \theta}\right)\right|=O[H(r) \log |2 \csc \theta|]$ uniformly in $0<|\theta|<\pi$. 
(iii) $S(r)=O[H(r)]$

For proof note that (i) $\Rightarrow$ (iii) by the first estimate in Theorem 4 , together with Theorem 5 (i). The implication (iii) $\Rightarrow$ (ii) follows from the two estimates in Theorem 4, together with Theorem 5 (i) and Theorem 6 (i). This is true because the inequality

$$
|A(u)| \leqq H(r) r^{\rho}(|x|-R)^{-\rho} \quad \text { for } \quad|x|<R, \quad|x-u|<R
$$

holds whenever $|A(u)| \leqq H(|u|)$ with $H \in M_{\rho}$. Thus we get a suitable value for $M$ in Theorem 5. Finally, (ii) $\Rightarrow(\mathrm{i})$, completing the circle of equivalence.

By Theorem 5 (ii) and (iv) we see that Theorem 7 also applies when the condition " $=O(H)$ " is replaced throughout by " $\in B_{\rho}$ " or " $\in B_{\rho}^{+},|\rho|<1$. But the assertion of uniformity requires $|\csc \theta|$ instead of $\log |2 \csc \theta|$, if by uniformity we mean that the dominating function $\phi(r)$ in (25) can be selected independently of $\theta$.

As a special case we can choose $H(r)=h(r) r^{\rho}$ where $h(r)$ is constant or tends to zero in such a way that $\mathrm{H} \in M_{\rho}$. Theorem 7 therefore applies when the condition $O(H)$ is replaced by $O\left(r^{\rho}\right)$ or $o\left(r^{\rho}\right)$, $|\rho|<1$. The choice $\rho=0$, leading to $O(1)$, or $o(1)$, gives an extension of Lindelöf's criterion to meromorphic functions of the type considered in Theorem 1. In this connection we should recall that the condition $A(u)=O[H(|u|)]$ can be replaced by the corresponding condition on

$$
F_{1}(r)=\frac{1}{2 \pi} \int_{-\pi}^{\pi} r^{-p} \log \left|F\left(r e^{i \theta}\right)\right| d \theta
$$

provided (26) holds for the function $A$.

Introduction of $F_{1}(r)$ shows an interesting contrast between the cases $p$ even, $p$ odd. In the first case $F_{1}(r)$ and $S(r)$ depend on $\Lambda_{o}$ only, and each is readily estimated in terms of the other. But in the latter case $F_{1}(r)$ involves $\Lambda_{o}$ only, while $S(r)$ involves $\Lambda_{e}$ only. Thus, the two terms are wholly independent. A somewhat different formulation of the content of Theorem 7 produces Tauberian theorems, relating the function

$$
F_{1}(r, \theta)=r^{-p} \log \left|F\left(r e^{i \theta}\right)\right|-S(r) \cos p \theta
$$

to $F_{1}(r)$. The Tauberian condition is (26) for $\dot{\phi}=A$, which is always verified when $F$ is entire.

These results can be extended as indicated in $\S 7$. For conditions of the type

$$
\left|A(-u)-D^{-}\right| \leqq H(|u|), \quad\left|A(u)-D^{+}\right| \leqq H(u), \quad u>0,
$$

we choose $G$ in (34) and obtain 


$$
\begin{aligned}
F_{1}(r, \theta)= & r^{-p} \log \left|F\left(r e^{i \theta}\right)\right| \\
& -\left[\pi D^{+} \operatorname{sgn} \theta+\left(D^{-}-D^{+}\right) \theta\right] \sin p \theta-\widetilde{A}(r) \cos p \theta,
\end{aligned}
$$

instead of (39). The resulting statements about $F_{1}(r, \theta)$ overlap with Theorem 2.

9. Equal right and left densities. We discuss the case $D^{-}=D^{+}$ in (40) and (41), for which the densities of combined zeros and poles are the same in each half plane. The main result is:

THEOREM 8. Let $|A(u)-D| \leqq H(|u|)$ where $H \in M_{\rho}, \quad|\rho|<1$, and define

$$
F_{1}(r, \theta)=r^{-p} \log \left|F\left(r e^{i \theta}\right)\right|-\pi D \sin p \theta \operatorname{sgn} \theta-\widetilde{A}(r) \cos p \theta .
$$

Then for any constant $C>2$ there is a constant $C_{1}$ such that:

(i) $\left|F_{1}(r, \theta)\right| \leqq C H(r)\left[\log |\csc \theta|+C_{1}\right] \quad 0<|\theta|<\pi$

(ii) $F_{1}(r, \theta) \leqq C H(r)\left[\log ^{+} \frac{1}{H(r)}+C_{1}\right]$ provided $F$ is entire, $p$ is odd, and $(1-p) A(u)$ is bounded below.

Moreover, if instead of $H \in M_{\rho}$ we have $H(r)$ decreasing and $r H(r)$ increasing for large $r$, the conclusions (i) and (ii) hold with an extra error term $2 H(r) \log ^{+} \frac{1}{H(r)}$.

We first consider the case $H \in M_{\rho}$. The preceding results are applied to $F(z)-z^{p} G(z)$ with $B(u)=D$, so that $G(z)=-\pi i D \operatorname{sgn} \theta$. By Theorem $5(i)$, the first estimate of Theorem 4 gives both parts of Theorem 8 in any region of the type $|\sin \theta| \geqq \delta>0$. We therefore use the second estimate of Theorem 4 , taking $|\sin \theta| \leqq \delta$ and $R=\delta|x|$. The positive constant $\delta$ is chosen so small that

$$
\left(1+\frac{1}{4} \eta\right)(1+\delta) \leqq\left(1+\frac{1}{3} \eta\right)
$$

and also, by (37), so small that $E(u)=A(u)-D$ satisfies

$$
|E(u)| \leqq\left(1+\frac{1}{4} \eta\right) H(r), \text { for }|x-u| \leqq R .
$$

The result of applying Theorem 4 is now

$$
\left|\log F(z)-z^{p} \widetilde{A}(r)-\pi i D z^{p} \operatorname{sgn} \theta-z^{p} E^{*}(z, R)\right| \leqq(\text { const }) H(r) r^{p} .
$$

We have used the fact that $\widetilde{E}(|x|)=\widetilde{A}(|x|)=\widetilde{A}(r)+O[H(r)]$. Since $E^{*}(z, R)$ can be estimated by (43) and Theorem 6(i), we obtain 


$$
\left|F_{1}(r, \theta)\right| \leqq\left(2+\frac{1}{2} \eta\right)\left(\log |\csc \theta|+C_{1}\right) H(r)
$$

where $C_{1}$ is constant. If $|\sin \theta|$ is small, this is less than the bound in Theorem 8 even with $C_{1}=0$. The inequality for other values of $\theta$ is ensured by choosing $C_{1}$ large enough.

The second assertion depends on the fact that $u A(u) \in K\left(D_{o}\right)$ for some constant $D_{0}$. Indeed, if the graph $s=\Lambda_{1}(u)$ is approximated within $\varepsilon$ by an increasing differentiable function $A_{\varepsilon}(u)$, the corresponding expression $A_{\varepsilon}$ satisfies

$$
\left[u A_{\varepsilon}(u)\right]^{\prime}-\left[u^{1-p} \Lambda_{\varepsilon}(u)\right]^{\prime} \geqq(1-p) u^{-p} \Lambda_{\varepsilon}(u)=(1-p) A_{\varepsilon}(u) .
$$

This gives $u A_{\varepsilon}(u) \in K\left(D_{o}\right)$ where $D_{o}$ is independent of $\varepsilon$, and hence $u A(u)$ has the same property. We have used the fact that $u^{1-p} \geqq 0$ for $p$ odd, and also the assumption that $(1-p) A(u)$ is bounded below.

Since $u A(u) \in K\left(D_{o}\right)$, evidently $u E(u) \in K\left(D_{o}+D\right)$. Writing $E^{*}$ as an abbreviation for $E^{*}(z, R)$, we note that

$$
z^{p} E^{*}=z^{p-1} z E^{*}=z^{p-1}[u E(u)]^{*}+O\left[r^{p} H(r)\right]
$$

by (35). If $0<s<R$ the interval of integration $(x-R, x+R)$ can be broken at the points $x-s$ and $x+s$. The integral over $(x-s$, $x+s$ ) is estimated by Theorem 6(ii) and the rest of the integral by (43). Thus,

$$
\operatorname{Re}[u E(u)]^{*} \leqq 2\left(D_{o}+D\right) s+2\left(1+\frac{1}{4} \eta\right) H(r)(|x|+R) \log \frac{R}{s} .
$$

We choose $s=\min [R, r H(r)]$, and recall that $|x|+R \leqq r(1+\delta)$. In view of (42) the result is

$$
\operatorname{Re}[u E(u)]^{*} \leqq 2\left(1+\frac{1}{3} \eta\right) r H(r) \log ^{+} \frac{1}{H(r)}+O[r H(r)] .
$$

By Theorem 6(i), the imaginary part of $[u E(u)]^{*}$ is $O[r H(r)]$. Hence, using (45),

$$
\operatorname{Re}\left(z^{p} E^{*}\right)=r^{p-1} \cos (p-1) \theta \operatorname{Re}[u E(u)]^{*}+O\left[r^{p} H(r)\right]
$$

and, taking real parts in (44),

$$
r^{p} F_{o}(r, \theta) \leqq \operatorname{Re}\left(z^{p} E^{*}\right)+O\left[r^{p} H(r)\right] .
$$

Since $|\sin \theta|$ is small and $p$ is odd, $\cos (p-1) \theta>0$. The sense of the inequality (46) is therefore preserved in (47), and we obtain the statement (ii).

The final assertion follows from 


$$
\frac{1}{r} \int_{0}^{r} H(u) d u \leqq-H(r) \log H(r)+O(H),
$$

and from a similar estimate for the expression (24), with a factor 2 in the principal term. The details are similar to those in [11].

To see the connection of Theorem 8 with results of the literature, let $p=1$ and define $h(r)=r H(r)$. The hypothesis is then

$$
|\Lambda(u)-D u| \leqq h(|u|)
$$

and the conclusion (ii) states

$$
\log |F(z)| \leqq x \int_{-r}^{r} \frac{\Lambda(u)}{u^{2}} d u+\pi D|y|+C h(r)\left[\log ^{+} \frac{r}{h(r)}+C_{1}\right]
$$

If $h(r)$ is increasing and $r^{-1} h(r)$ decreasing, $C$ is any constant larger than 4 , and if $r^{-1} h(r) \in M_{\rho}$ with $|\rho|<1$ then $C$ is any constant larger than 2. As shown by examples in [11], the values 2 and 4 cannot be improved.

Equation (49) refers to entire functions of genus 1. For meromorphic functions the result is given by Theorem 8(i), and in particular, (49) holds as an equality, with $C$ replaced by a bounded function of $r$ and $\theta$, in any region of the type

$$
|\sin \theta| \geqq(\text { const) } H(r) .
$$

This is evident by comparing the forms of the error in (i) and (ii).

When $F$ is even the integral on the right of (49) is 0 . Hence, (49) extends and sharpens several results of Boas and the author [3], [12], [11]. If

$$
\lambda_{n+1}-\lambda_{n} \geqq c>0,
$$

as is assumed by Boas, we can also extend his estimate

$$
\log \left|F^{\prime}\left(\lambda_{n}\right)\right| \geqq-C_{2} h(r) \log \left[\frac{r}{h(r)}\right]-C_{3} h(r)
$$

to the more general functions being considered here. With

$$
W(x, \lambda)=\left(1-\frac{x}{\lambda}\right) e^{x / \lambda}, \quad \lambda \equiv \lambda_{n},
$$

we have

$$
\frac{F(x)-F(\lambda)}{x-\lambda}=-\frac{F(x)}{W(x, \lambda)} \frac{e^{x / \lambda}}{\lambda}=F_{1}(x) \lambda^{-1} e^{x / \lambda} \rightarrow e \lambda^{-1} F_{1}(\lambda)
$$

as $x \rightarrow \lambda$, where $F_{1}$ is defined by the equation. When $x=\lambda$ the function $F_{1}(x)$ satisfies the conditions needed in Theorem 6(iii). This 
latter result can therefore take the part that was formerly taken by Theorem 6(ii); and a simplified version of the argument leading to Theorem 8 now gives (50).

10. Oriented products. If $A(u)=0$ for $u>0$, the decomposition into even and odd parts is unnecessary, and the appropriate conditions on $A$ are, as a rule, such that $\widetilde{A}(r)$ is an error term rather than a principle term. But despite this increased simplicity, the desired estimates are not special cases of the foregoing. The trouble is that the previous error terms are large on the positive real axis, whereas they should now be small.

To discuss these matters define

$$
A^{0}(r)=\int_{0}^{r} \frac{|A(-u)|}{u} d u+r \int_{r}^{\infty} \frac{|A(-u)|}{u^{2}} d u
$$

and let $\phi^{0}(r)$ be given by (51) with $A(-u) \equiv \phi(u)$, for any function $\phi$. The expression (51) is an error term analogous to those in (19). Though (51) does not follow from (19) by putting $m=0$, we have

$$
\phi^{2}(r) \leqq \phi^{1}(r) \leqq \phi^{0}(r) \leqq \tilde{\phi}(r)+\phi^{1}(r)
$$

and hence, $\phi^{0}(r)$ can be estimated by Theorem 5. Since $\phi^{0}(r)$ is increasing, it is automatically in $B_{\rho}^{+}$if in $B_{\rho}$.

If $\phi$ is a function of $r$ the statement " $\phi \in L_{\rho}$ " means that $\phi$ is positive and integrable, and

$$
\lim _{r \rightarrow \infty} \frac{\phi(t r)}{\phi(r)}=t^{\rho}, \quad t>0 .
$$

A function of $u$ belongs to $L_{\rho}$ if its even and odd parts do when regarded as functions of $r$. As was the case for $B_{\rho}$, here too interest centers about the behavior as $r \rightarrow \infty$. Convergence of the integrals near 0 is assured for the functions with which we are concerned, and is not emphasized in the sequel.

It is known [6] that the functions satisfying (53) can be represented in the form

$$
\dot{\phi}(r)=r^{\rho} p(r) \exp \int_{1}^{r} \frac{q(t)}{t} d t
$$

where $\lim p(r)=p_{o}>0$ exists and $\lim q(r)=0$ as $r \rightarrow \infty$. This shows that the convergence (53) is uniform on $\delta \leqq t \leqq 1 / \delta$. As is also known [9], the class $L_{\rho}$ is a slight generalization of the class $\left\{r^{\rho(r)}\right\}$, where $\rho(r)$ is a Lindelöf proximate order with limit $\rho$. Using this fact, or (54), we obtain 


$$
\left\{\begin{array}{l}
\phi \in L_{\rho} \Rightarrow \tilde{\phi}(r) \sim \rho^{-1} \phi(r) \text { for } \rho>0, \\
\phi \in L_{\rho} \Rightarrow \phi^{m}(r) \sim 2 m\left(m^{2}-\rho^{2}\right)^{-1} \phi(r) \text { for }|\rho|<m .
\end{array}\right.
$$

These serve the same purpose for the class $L_{\rho}$ as was served by Theorem 5 for $B_{\rho}$ and $M_{\rho}$.

Theorem 9. Let $A(u)=0$ for $u>0$. Then for $|\theta|<\pi$

$$
\left|z^{-p} \log F(z)\right| \leqq\left|\sec \frac{1}{2} \theta\right| A^{0}(r),
$$

and for $x+R \leqq 0$,

$$
\left|z^{-p} \log F(z)-A^{*}(z, R)\right| \leqq 2 \frac{|x|}{R} A^{0}(|x|) .
$$

Also if $A(-u) \sim H(u) \in L_{\rho}, 0<\rho<1$, then

$$
\left|z^{-p} \log F(z)-H(r) e^{i \rho} \pi \csc \pi \rho\right| \leqq \eta H(r) \log \left(2 \sec \frac{1}{2} \theta\right)
$$

where $\eta \rightarrow 0$ uniformly in $|\theta|<\pi$ as $r \rightarrow \infty$.

With $k_{3}=z /(z+u)$ as before, we set $k_{5}=1-k_{3}$ and note that each function satisfies

$$
\left|k_{i} \cos \frac{1}{2} \theta\right| \leqq 1 \text { for } u>0 .
$$

The first assertion follows from this and (5). The second follows from

$$
\begin{aligned}
z^{-p} \log F(z)-A^{*}(z, R)= & \int_{-\infty}^{x-R} \frac{A(u)}{u^{2}} k_{6} d u+\int_{x-R}^{x} \frac{A(u)}{u} d u \\
& +\int_{x+R}^{0} \frac{A(u)}{u} k_{7} d u+\int_{x}^{x+R} \frac{A(u)}{u} d u
\end{aligned}
$$

where $k_{6}=z u /(z-u)$ and $k_{7}=z /(z-u)$. We use the fact that

$$
\left|k_{6}\right| \leqq|x-R| \frac{|x|}{R} \leqq 2|x| \frac{|x|}{R}, \quad\left|k_{7}\right| \leqq \frac{|x|}{R}
$$

on the relevant intervals.

For the third statement there is no loss of generality in taking $A(-u)=H(u)$. According to (33) and (5)

$$
z^{-p} \log F(z)-H(r) e^{i \rho} \pi \csc \pi \rho=\int_{0}^{\infty}\left[H(u)-H(r)\left(\frac{u}{r}\right)^{\rho}\right] \frac{z d u}{u(z+u)} .
$$

If $\delta$ is a small positive number, the integral from 0 to $\delta r$ is uniformly 
$O\left[\delta^{\rho} H(r)\right]$ by (55) and (53). Similarly, the integral from $r / \delta$ to $\infty$ is uniformly $O\left[\delta^{1-\rho} H(r)\right]$.

For the integral from $\delta r$ to $r / \delta$ the substitution $u=r t$ gives an upper bound of form

$$
H(r) \int_{\delta}^{1 / \delta}\left|\frac{H(t r)}{H(r)}-t^{\rho}\right| \frac{d t}{t|\xi+t|}, \quad \xi=e^{i \theta} .
$$

Since the convergence (53) is uniform, the proof of Theorem 9 is complete if

$$
\int_{\delta}^{1 / \delta} \frac{d t}{t|\xi+t|} \leqq \text { (const) } \log 2 \sec \frac{1}{2} \theta,
$$

$\delta$ being fixed. But (56) is easily verified by (27).

The first two parts of Theorem 9 correspond to Theorem 4, and hence, the discussion of $A^{*}$ and of comparison applies without change. For example, let $\alpha, \beta$ and $\rho$ be constants between 0 and 1 , and for $u>0$ suppose $\left|A(-u)-u^{\rho}\right| \leqq H(u)$, where $u^{-\alpha} H(u)$ increases and $u^{\beta-1} H(u)$ decreases. Then

$$
\left|\log F(z)-\pi z^{p+\rho} \csc \pi \rho\right| \leqq r^{p} H(r)\left(\frac{1}{\alpha}+\frac{1}{\beta}\right) \sec \frac{1}{2} \theta
$$

for $|\theta|<\pi$, as is seen by use of the comparison function (33) in the first assertion of Theorem 9. A sharper result, involving $\log \sec \theta / 2$, follows from the second assertion. The third statement of Theorem 9 sharpens and extends the classical result of Valiron [9].

Instead of the particular function $u^{\rho}$ one can introduce an arbitrary comparison function $B(u)$. The resulting development yields an alternative form of Theorem 2, in which the given condition on $A(u)$ is replaced by

$$
\lim _{r \rightarrow \infty}\left[\frac{A(r u)}{B(r u)}-\frac{A(r / u)}{B(r / u)}\right]=0, \quad \frac{A(u)}{B(u)}=O(1) \quad(u \neq 0) .
$$

11. Elementary remarks on completeness. The function $A^{*}$ introduced in the preceding discussion has an interesting connection with the problem of completeness of complex exponentials. A set $\left\{e^{i \lambda_{n} x}\right\}$ has completeness length $I$ if it is complete on every interval of length less than $I$, and on no larger interval. In other words, the completeness length is $I$ if the condition

$$
G(z)=\int_{-a}^{a} f(t) e^{i z t} d t=0 \text { at } z=\lambda_{n}
$$

implies that $f$ is equivalent to 0 , when $a<I / 2$, and has a nontrivial solution $f$ when $a>I / 2$. It is well known that $I$ is independent of 
the class $L^{p}$ to which $f$ belongs. We therefore take $f \in L^{2}$, so that the Paley-Wiener representation theorem can be used. If the set is complete on no interval we set $I=0$, and if it is complete on every interval, then $I=\infty$.

To distinguish the completeness interval for $\exp \left(i \lambda_{n} x\right)$ from that for $\exp \left(i \mu_{n} x\right)$ we write $I(\lambda)$ or $I(\mu)$, as the case may be. As elsewhere in this paper, $\Lambda_{1}$ is the counting function for the $\lambda^{\prime} s$ and $\Lambda_{2}$ for the $\mu^{\prime} s$, and $\Lambda=\Lambda_{1}-\Lambda_{2}$. The assumption that $\lambda \neq 0$ and $\mu \neq 0$ involves no loss of generality, because any finite number of $\lambda^{\prime} s$ or $\mu^{\prime} s$ can be altered without affecting the completeness.

It is convenient to define

$$
c=\int_{-\infty}^{\infty} \frac{\Lambda(u)}{u^{2}} d u, \quad \Lambda^{\infty}(x)=x \int_{|x|}^{\infty} \frac{\Lambda(u)+\Lambda(-u)}{u^{2}} d u .
$$

The notation $\Lambda^{\infty}$ suggests a function that describes the behavior of $\Lambda$ near $\infty$. This contrasts with the function

$$
\Lambda^{*}(x, R)=\int_{x-R}^{x+R} \frac{\Lambda(u)}{x-u} d u,
$$

which is a measure of local behavior.

Theorem 10 (lemma). If $\Lambda \in B_{1}$ and $p=1$, then

$$
\log \left|e^{-c x} F(x)\right|+\Lambda^{\infty}(x)-\Lambda^{*}(x, R) \in B_{1}^{+}
$$

where $R$ is any positive measurable function of $x$ such that $R / x$ and $x / R$ remain bounded as $|x| \rightarrow \infty$.

If $F_{1}(z)$ is any function with zeros $\lambda_{n}$ and poles $\mu_{n}$, by using Jensen's theorem as in [7] we get

$$
\log \left|F_{1}(x)\right|-\Lambda^{*}(x, R)=\frac{1}{2 \pi} \int_{0}^{2 \pi} \log \left|F_{1}\left(x+R e^{i \theta}\right)\right| d \theta .
$$

The choice $F_{1}(z)=e^{-c z} F(z)$ gives an alternative form of this lemma. For proof observe that the function $A(u)=\Lambda(u) / u$ satisfies

$$
u A^{1}(u) \in B_{1}^{+}, \quad u A^{2}(u) \in B_{1}^{+}
$$

by Theorem 5 (iv). Also Theorem 5 (ii) gives

$$
x A^{*}(x, R)-\Lambda^{*}(x, R) \in B_{1}^{+}
$$

provided $R=O(|x|)$. Theorem 10 now follows from Theorem 4, after multiplication by $x$.

To illustrate the use of this result suppose $I(\mu)<\infty$ and let $G(z)$ be the function (58), with a slightly larger than $I(\mu) / 2$. When 
$G(\mu)=0$, partial integration gives

$$
\frac{1}{\mu-x} \int_{-a}^{a} f(t) e^{i x t} d t=\int_{-a}^{a} f_{1}(t) e^{i x t} d t
$$

where

$$
f_{1}(t)=i e^{-i \mu t} \int_{-a}^{t} e^{i \mu w} f(w) d w .
$$

Hence, $\left|f_{1}(t)\right|$ can be estimated by the integral of $|f(w)|$, and repetition gives a multiple integral just like the remainder term in Taylor's series. If

$$
P(x)=\Pi \frac{1}{x-\mu_{n}} \quad(N \text { factors })
$$

and $G$ is suitably normalized, it follows that

$$
|P(x) G(x)| \leqq \frac{(2 a)^{N}}{N !}
$$

On the other hand, for $S \geqq 0$

$$
\Lambda^{*}(x, S)=\Lambda_{1}{ }^{*}(x, S)-\Lambda_{2}{ }^{*}(x, S) \leqq-\Lambda_{2}{ }^{*}(x, S),
$$

since $A_{1}(u)$ is nondecreasing. By partial integration

$$
-\Lambda_{2}(x, S)=\log |P(x)|+N \log S
$$

where $P(x)$ is the product over all $\mu$ on the interval $(x-S, x+S)$, and $N$ is the number of $\mu$ 's on this interval. Since $(2 a S)^{N} / N !<e^{2 a S}$, the foregoing inequalities give

$$
\Lambda^{*}(x, S)+\log |G(x)| \leqq 2 \alpha S .
$$

When $\Lambda \in B_{1}$, Theorem 10 gives

$$
\log \left|e^{-c x} F(x) G(x)\right| \leqq \Lambda^{*}(x, R)-\Lambda^{*}(x, S)+2 a S-\Lambda^{\infty}(x)
$$

apart from a function of class $B_{1}^{+}$.

If $\phi(x) \in B_{1}^{+}$, there is an entire function $E(z)$, of arbitrarily small type, such that $|E(x)| \leqq \exp [-\phi(|x|)]$. (A simple proof of this well known result is given in [10] and also in [12].) Since (10) shows that $\log |F(i y)|=o(|y|)$ when $A \in B_{1}$, we conclude that $I(\lambda) \leqq I(\mu)$, if the function on the right of (61) admits an upper bound $\phi(x) \in B_{1}^{+}$. Symmetry of the hypothesis then gives $I(\lambda)=I(\mu)$.

A convenient way to handle the term $\Lambda^{\infty}(x)$ is to assume

$$
\int_{1}^{\infty} \frac{|\Lambda(u)+\Lambda(-u)|}{u^{2}} \log u d u<\infty .
$$


In this case the function $\phi(r)=|\Lambda(r)+\Lambda(-r)|$ satisfies $\phi^{1} \in B_{1}^{+}$by Theorem 5(v). Since $\left|\Lambda^{\infty}(r)\right|<\phi^{1}(r)$, we conclude that $\Lambda^{\infty} \in B_{1}^{+}$.

It is also true that $\Lambda^{\infty} \in B_{1}^{+}$if $\Lambda \in B_{1}$ and if (62) holds with $\Lambda(u)+\Lambda(-u)$ replaced by its mean value $\bar{\Lambda}(u)$,

$$
\bar{\Lambda}(u)=\frac{1}{u} \int_{0}^{u}[\Lambda(t)+\Lambda(-t)] d t .
$$

Indeed, the fact that $\Lambda \in B_{1}$ makes $\bar{\Lambda}(u)=o(u)$, and hence we can express $\Lambda^{\infty}$ in terms of $\bar{A}$ by partial integration. The conclusion follows from Theorem 5 as before. Whenever $\Lambda^{\infty} \in B_{1}^{+}$, this term can be dropped from (61). (For symmetric sequences, $A^{\infty}=0$.)

The foregoing discussion gives the following simple result:

THEOREM 11 (remark). We have $I(\lambda)=I(\mu)$ whenever

$$
\frac{\log r}{r} \int_{-r}^{r} \Lambda(u) d u \in B_{1} \text { and }\left(\int_{-r}^{r}|\Lambda(u)| d u\right)^{1 / 2} \in B_{1} .
$$

For proof, by inspection

$$
\left|\Lambda^{*}(x, R)-\Lambda^{*}(x, S)\right| \leqq \frac{1}{S} \int_{x \rightarrow R}^{x+R}|\Lambda(u)| d u .
$$

If $S$ is chosen to be the square root of the integral we are led to the second condition in Theorem 11. The first condition follows from the remarks made in connection with (62). It should be observed that the conditions are independent, in the sense that neither follows from the other, even if $\Lambda(u)$ is replaced by $|\Lambda(u)|$.

The interest of Theorem 11 lies partly in the fact that the proof is so elementary, and partly in the fact that no regularity is assumed for the individual sequences $\left\{\lambda_{n}\right\}$ and $\left\{\mu_{n}\right\}$. Thus, $\left\{\lambda_{n}\right\}$ might have density 0 and $I(\lambda)=\infty$ without invalidating the conclusion.

By the Schwarz inequality the second condition of the theorem holds if

$$
\frac{\phi(r)}{r} \int_{-r}^{r}|\Lambda(u)| d u \in B_{1}
$$

where $\phi$ is any positive function such that

$$
\int_{r}^{\infty} \frac{d r}{r \phi(r)}<\infty
$$

If $\phi(r) / r$ is decreasing, an integration by parts shows that this new condition can be replaced by

$$
\phi(|u|)|\Lambda(u)| \in B_{1} .
$$


In particular, we can take $\phi(r)=\log r(\log \log r)^{a}$ with $a>1$. Since the choice $\phi(r)=\log r$ is already sufficient to ensure the first condition of Theorem 11, $I(\lambda)=I(\mu)$ follows with no further hypothesis. In the special case in which $\left\{\lambda_{n}\right\}$ is symmetric and $\Lambda_{2}(u)=D u$ the result is a sharpened form of a criterion of Koosis ([8], Th. 11).

12. Additional remarks on completeness. Apart from the Paley-Wiener theorem, the foregoing discussion from beginning to end uses nothing more elaborate than integration by parts. We now mention a stronger result that follows from the deep theorem of Beurling and Malliavin [1].

If the two functions

$$
\Lambda(x) \text { and } \Lambda^{*}(x, R)-\Lambda^{\infty}(x)
$$

of Theorem 10 both belong to $B_{1}$, the result [1] gives $I(\lambda)=I(\mu)$. The same holds if the fuction on the right of (61) admits an upper bound in $B_{1}$. We use this latter observation to establish. ${ }^{1}$

Theorem 12. If $\Lambda^{\infty}(x) \in B_{1}$ and $\Lambda(x) \log \log |x| \in B_{1}$, then $I(\lambda)=I(\mu)$.

The right side of $(61)$ is $\phi(x)-\Lambda^{\infty}(x)$, where

$$
\phi(x)=2 a S-\int_{S}^{R} \frac{\Lambda(x+t)-\Lambda(x-t)}{t} d t .
$$

We form a sequence with $x_{n}=2^{n}$ and $y_{n}$ so that $\left(y_{n}, y_{n+1}\right)$ is the middle third of the interval $\left(x_{n}, x_{n+1}\right)$. On the interval $\left(x_{n}, x_{n+1}\right)$ it is convenient to define

$$
R(x)=R_{n}=\frac{1}{3} x_{n}, \quad S(x)=S_{n}=\frac{1}{n^{2}} R_{n} .
$$

Since $x_{n}=y_{n}-R_{n}<y_{n+1}+R_{n}=x_{n+1}$, for $S_{n} \leqq t \leqq R_{n}$ it follows that

$$
\int_{y_{n}}^{y_{n+1}}|\Lambda(x+t)-\Lambda(x-t)| d x \leqq 2 \int_{x_{n}}^{x_{n+1}}|\Lambda(x)| d x \text {. }
$$

Hence by (63)

$$
\int_{y_{n}}^{y_{n+1}}|\dot{\phi}(x)| d x \leqq a S_{n} x_{n}+2 \log \frac{R_{n}}{S_{n}} \int_{x_{n}}^{x_{n+1}}|\Lambda(x)| d x .
$$

Dividing by $x_{n}^{2}$ and summing on $n$, we conclude that

$$
\int_{E} \frac{|\phi(x)|}{x^{2}} d x<\infty
$$

${ }^{1}$ Note added in proof (July 1967). Recently published work of Beurling and Malliavin shows that $\Lambda(x) \in B_{1}$ is actually sufficient. 
where $E$ is the set of all the middle thirds. A similar calculation using a different sequence $\left\{x_{n}\right\}$ gives the result for the left-hand thirds, and likewise for the remaining thirds. Repeating the argument for $x<0$, we find that $\phi(x) \in B_{1}$, and hence $I(\lambda) \leqq I(\mu)$. Symmetry gives $I(\lambda)=I(\mu)$.

The above choice of $S_{n}$ leads to a simple result but is not optimum. If $S_{n}$ is chosen so as to minimize the estimate, the needed condition is found to be

$$
I_{n} \rightarrow 0, \quad \sum I_{n} \log ^{+} \frac{1}{I_{n}}<\infty, \text { where } \quad I_{n}=\int_{x_{n}}^{x_{n+1}} \frac{|\Lambda(x)|}{x^{2}} d x .
$$

This is sharper than the former condition $\sum I_{n} \log n<\infty$.

The foregoing results do not assume that either sequence $\left\{\lambda_{n}\right\}$ or $\left\{\lambda_{n}\right\}$ has a density. However, the special case $\Lambda_{2}(u)=D u$, where $D$ is a positive constant, gives added insight into the function $\Lambda^{*}$. We then have

$$
\Lambda(u)=\Lambda_{1}(u)-D u, \quad \Lambda^{*}(x, R)=\Lambda_{1}^{*}(x, R)+2 D R
$$

and the criterion of Koosis [7] can be stated as follows: If the set $\left\{e^{i \lambda_{n} x}\right\}$ is not complete on an interval of length $2 \pi D$, there is a function $\phi(x) \in B_{1}$, independent of $R$, such that

$$
-\Lambda^{*}(x, R) \leqq \phi(x) \text {. }
$$

On the other hand, assuming $\Lambda \in B_{1}$ and $\Lambda^{\infty} \in B_{1}$, our results show that $I(\lambda) \leqq 2 \pi D$ if

$$
\Lambda^{*}(x, R) \leqq \phi(x)
$$

where $\phi \in B_{1}$. Here the condition is needed not for all $R$ but only for $R$ restricted as in Theorem 10 .

A set $\left\{e^{i \lambda_{n} x}\right\}$ is said to be exact if it is complete on some interval but becomes incomplete when one term $e^{i \lambda x}$ is removed. The set has finite (positive or negative) excess if it becomes exact upon removal or adjunction of finitely many terms. We now establish:

THEOREM 13 (remark). Let $\left\{e^{i \lambda \cdot n^{x}}\right\}$ have finite excess on an interval of length $2 \pi D$ and suppose $\Lambda(u) \equiv \Lambda_{1}(u)-D u \in B_{1}$. Then $\Lambda^{\infty} \in B_{1}^{+}$. Also, if $f(t)$ is not equivalent to 0 and is orthogonal to all but a finite number of functions $e^{i \lambda_{n} t}$, the function

$$
G(z)=\int_{-\pi D}^{\pi D} f(t) e^{i z t} d t
$$

satisfies 


$$
\frac{1}{2 \pi} \int_{0}^{2 \pi} \log \left|G\left(x+R e^{i \theta}\right)\right| d \theta-2 D R \in B_{1}^{+}
$$

when $R$ is restricted as in Theorem 10 .

Without loss of generality we can assume that $\left\{\lambda_{n}\right\}$ consists precisely of the zeros of $G(z)$, and that $G(0)=1$. As far as the form of $G$ is concerned, we shall use only the fact that $G(z)$ satisfies

$$
|G(z)| \leqq e^{\pi D|y|+\phi(|x|)}
$$

where $\phi \in B_{1}^{+}$. The Hadamard factorization theorem then shows that the function $G$ is identical with the function $e^{-c z} F(z)$ of Theorem 10, with $\Lambda_{2}(u)=D u$. By Jensen's theorem

$$
\log |G(x)|-\Lambda_{1}^{*}(x, R)=\frac{1}{2 \pi} \int_{0}^{2 \pi} \log \left|G\left(x+R e^{i \theta}\right)\right| d \theta .
$$

Since $\Lambda^{*}(x, R)=\Lambda_{1}^{*}(x, R)+2 D R$, the inequality (64) together with the above gives

$$
\log |G(x)|-\Lambda^{*}(x, R) \leqq \dot{\phi}(|x|) .
$$

Theorem 10 now shows that $\mathscr{A}^{\infty}(x)$ admits a lower bound in $B_{1}^{+}$for $-\infty<x<\infty$, and since $\Lambda^{\infty}$ is odd, we conclude that $\Lambda^{\infty} \in B_{1}^{+}$. Applying Theorem 10 again gives the second assertion.

13. Examples concerning entire functions. The foregoing analysis distinguishes rates of growth specified by

$$
h(r), \quad h(r) \log \frac{r}{h(r)}, \quad h(r) \log r .
$$

Also the classes $B_{1}$ and $B_{1}^{+}$are distinguished. We give examples to show that these distinctions do not result from inadequacy of the analysis, but are essential.

Throughout the discussion

$$
\Lambda(u)=\Lambda_{1}(u)-D u
$$

where $A_{1}$ is the counting function for the real sequence $\left\{\lambda_{n}\right\}$ and $D$ is a constant between 0 and 1 . The condition $D<1$ enables us to construct examples with simple zeros at the integers only, but is otherwise irrelevant.

We use $h$ to denote a function which is continuous, positive, and even, and satisfies the conditions

$$
h(r)=o(r), \quad r^{-1} h(r) \in M_{p}
$$


for some $\rho, 0<\rho<1$. (The latter condition could be considerably weakened.)

ExAMPLE 1. Let $|\Lambda(u)| \leqq h(|u|)$ and let

$$
G(z)=e^{-c z} \Pi\left(1-\frac{z}{\lambda_{n}}\right) e^{z / \lambda_{n}}
$$

where the constant $c$ is chosen so that $G(|x|)=o(|x|)$. Then if $h(r) \log r \in B_{1}$ we have $\log ^{+}|G(x)| \in B_{1}^{+}$. But if $h(r) \log r$ is not in $B_{1}$ there is a function $G$ of the above type, with simple zeros at the integers only, such that $\log ^{+}|G(x)|$ is not in $B_{1}$.

The example depends on the fact that, under the given hypothesis,

$$
\log \left|e^{-c x} F(x)\right|=\Lambda^{*}(x, R)-\Lambda^{\infty}(x)+E(x),
$$

where the error term $E$ satisfies

$$
R(1-\rho)|E(x)| \leqq \text { (const) }|x| h(|x|)
$$

for $R \leqq|x|$ and $|x| \gg 1$. This follows as in the proof of Theorem 10 . Since $\Lambda \in K(D)$ an easy argument, similar to others in this paper, gives

$$
\Lambda^{*}(x, R) \leqq 2 h(r) \log \frac{r}{h(r)}, \quad r=|x|,
$$

apart from terms of lower order. The dominant term is therefore $\Lambda^{\infty}$, in general. We now choose $A$ so that

$$
\Lambda(r)+\Lambda(-r) \doteq h(r),
$$

using the fact that, if $h$ is differentiable, $h^{\prime}(r)=o(1)$ as $r \rightarrow \infty$, and hence $h^{\prime}(r)<1-D$.

As suggested by this example, quite generally the behavior of $G$ is dominated by $\Lambda^{\infty}$ on the real axis, unless some special condition is imposed. From now on we assume that $F$ is even, so that $A^{\infty}=c=0$, and

$$
F(z)=\Pi\left(1-\frac{z^{2}}{\lambda_{n}^{2}}\right)
$$

The further construction of examples is based on $\Lambda^{*}$ rather than $\Lambda^{\infty}$. To simplify the statements, we say that the even function $F$ belongs to $h$ if $h$ has the above-described properties, $F$ is given by the foregoing canonical product, and the function $\Lambda(u)=\Lambda_{1}(u)-D u$ satisfies $|\Lambda(u)| \leqq h(u)$ for $u>0$. The statement that $F$ has integral zeros means that all the zeros $\lambda_{n}$ are integers, and are simple; that is, no $\lambda_{n}$ is repeated. By thus restricting the class of functions, we give 
more content to our examples.

EXAmple 2. If $h(r) \log r / h(r) \in B_{1}$, then $\log ^{+}|F(x)| \in B_{1}^{+}$for every even function $F$ belonging to $h$. But if $h(r) \log r / h(r)$ is not in $B_{1}$ then there is an even function $F$ with integer zeros, belonging to $h$, such that $\log ^{+}|F(x)|$ is not in $B_{1}^{+}$.

If $r>e h$, then $h \log r / h$ is increasing, and hence the positive statement follows from the proof of Example 1. In the course of proving the negative statement we shall also establish:

EXAMPLE 3. There is an even entire function of arbitrarily small type, with integral zeros, such that $\log |F(x)|$ is in $B_{1}$ but $\log ^{+}|F(x)|$ is not in $B_{1}^{+}$.

Results of this sort are established by the following observation: Let $x_{n}=a^{n}$ be any geometric sequence with $a>1$. Then if $\phi>0$ and $\phi \in M_{\rho}$ for some $\rho$, the conditions

$$
\int_{1}^{\infty} \frac{\phi(r)}{r^{2}} d r<\infty \quad \text { and } \quad \sum \frac{\phi\left(x_{n}\right)}{x_{n}}<\infty
$$

are equivalent. We omit the routine proof.

Let $h$ be given with $h \log r / h$ not in $B_{1}$, but let $h \in B_{1}$, as can be assumed by diminishing $h$. Form a sequence

$$
x_{n}=e^{n}, \quad y_{n}=e^{n+1 / 2}, \quad n \geqq 1
$$

so that $y_{n-1}<x_{n}<y_{n}$. The point sets $\left\{x_{n}\right\}$ and $\left\{y_{n}\right\}$ together form a geometric sequence with ratio $\sqrt{e}$. We construct $F$ belonging to $h$ with integer zeros as follows: The graph of $\Lambda(u)$ for increasing $u>0$ follows alternately the curves given by

$$
h(u), \quad D\left(x_{n}-u\right), \quad-h(u), \quad(1-D)\left(u-y_{n}\right) .
$$

Here the word "follows" means that the graph is within 1, say, of the designated path. Since $F$ is even, $\Lambda(-u)=-\Lambda(u)$, completing the definition. The precise behavior for small $u$ is not important.

Since $\Lambda^{\infty}=0$, we have

$$
\log |F(x)|=\Lambda^{*}(x, R)+O[h(x)], \quad x>0
$$

when $R / x$ and $x / R$ are bounded. The choice $R=(1 / 3) x_{n}$ gives

$$
\log \left|F\left(x_{n}\right)\right|=2 h\left(x_{n}\right) \log \frac{x_{n}}{h\left(x_{n}\right)}+O\left[h\left(x_{n}\right)\right] .
$$

Hence, if $\phi(x)$ is any monotone majorant of $\log ^{+}|F(x)|$ and $x$ is large, 


$$
9 \int_{x_{n}}^{x_{n+1}} \frac{\phi(x)}{x^{2}} d x \geqq \frac{2 h\left(x_{n}\right)}{x_{n}} \log \frac{x_{n}}{h\left(x_{n}\right)} \geqq \int_{x_{n}}^{x_{n+1}} \frac{h(x)}{x^{2}} \log \frac{h(x)}{x} d x .
$$

The second inequality follows from the fact that the integrand is monotonic for $e h(x)<x$, and shows that $\log ^{+}|F(x)|$ is not in $B_{1}^{+}$. This completes the proof of Example 2.

We now show that $\log ^{+}|F(x)| \in B_{1}$. The fact that $\log ^{-}|F(x)| \in B_{1}$ and hence $\log |F(x)| \in B_{1}$, follows from known properties of entire functions.

It is convenient to write $p \prec Q$ whenever

$$
P \leqq c_{1} Q+c_{2} h, \quad c_{1}, c_{2} \text { const } .
$$

If $y_{n-1}<x<y_{n}$, as now assumed, we have

$$
\log ^{+} F(x) \prec h(x) \log \frac{x}{h(x)}
$$

and also

$$
\log ^{+} F(x) \prec \int_{0}^{x_{n}-h} \frac{h(u)}{x-u} d u-\int_{x_{n}+h}^{2 x_{n}} \frac{h(u)}{x-u} d u
$$

where $h=h\left(x_{n}\right)$. The inequality (66) gives

$$
\int_{\left|x-x_{n}\right| \leqq 2 h} \frac{\log ^{+} F(x)}{x^{2}} d x \leqq \frac{h^{2}\left(x_{n}\right)}{x_{n}^{2}} \log \frac{x_{n}}{h\left(x_{n}\right)}
$$

apart from terms of lower order. Since $x_{n}=e^{n}$, the sum on $n$ can be estimated by an integral involving $e^{t}$. The change of variable $u=e^{t}$ gives

$$
\int^{\infty} \frac{h(u)}{u^{2}} \frac{h(u)}{u} \log \frac{u}{h(u)} d u<\infty
$$

If $x=x_{n}+t$, and $|t| \geqq 2 h$, a short calculation based on (67) gives

$$
\log ^{+} F(x) \prec h\left(x_{n}\right) \log \frac{x_{n}^{2}-t^{2}}{t^{2}} .
$$

We have used the fact that $h(u)$ can be replaced by $h\left(x_{n}\right)$ and that

$$
t^{2} \geqq t^{2}-h^{2} \geqq \frac{3}{4} t^{2}
$$

If (68) is integrated from $t=h$ to $t=x_{n} / \sqrt{2}$, and doubled to account for the interval $\left(-x_{n} / \sqrt{2},-h\right)$, we conclude that

$$
\int_{I_{n}} \log ^{+}|F(x)| d x \prec x_{n} h\left(x_{n}\right)
$$


where $I_{n}$ is the part of $\left(y_{n-1}, y_{n}\right)$ for which $\left|x-x_{n}\right| \geqq 2 h$. This gives

$$
\int_{I_{n}} \frac{\log ^{+} F(x)}{x^{2}} d x \prec \frac{h\left(x_{n}\right)}{x_{n}} .
$$

Summing on $n$ as before and setting $u=e^{t}$, we get

$$
\int^{\infty} \frac{H(u)}{u^{2}} d u<\infty \text {. }
$$

This completes the construction of Example 3.

The function satisfies the additional condition $\Lambda(u) \in B_{1}^{+}$. Hence, this condition does not ensure $\log ^{+}|F(x)| \in B_{1}^{+}$. On the other hand the condition

$$
\Lambda(u) \log \log |u| \in B_{1}
$$

ensures $\log |F(x)| \in B_{1}$ by an argument similar to that in Theorem 12 . We shall show that the factor $\log \log |u|$ cannot be dropped:

EXAMPLE 4. There is an even entire function with integral zeros, of type $\pi D$, such that $\Lambda(u) \in B_{1}$, but $\log ^{+}|F(x)|$ is not in $B_{1}$.

Form a sequence $x_{n}=4^{n}$ and let $\Lambda(u)$ follow alternately lines of slopes

$$
0, \quad 1-D, \quad-D, \quad 0
$$

so that the graph forms a triangle above the axis, which lies wholly to the left of $x_{n}$ and ends at $x_{n}$. If the area of the triangle is $J_{n}$, we require $\sum x_{n}^{-2} J_{n}<\infty$, so that $\Lambda(u) \in B_{1}$. The base of the triangle is $d \sqrt{J_{n}}$, where $d$ is a positive constant and, hence, the base is $o\left(x_{n}\right)$ as $n \rightarrow \infty$. There is therefore no interference between adjacent triangles.

For $x_{n}<x<(3 / 2) x_{n}$ and $R=x_{n}$ we have

$$
\Lambda^{*}(x, R) \geqq \frac{J_{n}}{x-x_{n}+d V \overline{J_{n}}},
$$

since the value of the integral would be diminished if the whole of the area $J_{n}$ were located at $x_{n}-d \sqrt{J_{n}}$. Hence,

$$
\int_{x_{n}}^{3 / 2 x_{n}} \Lambda^{*}(x, R) d x \geqq J_{n} \log \frac{x_{n}}{\sqrt{J_{n}}}
$$

apart from terms of lower order. Applying Theorem 10, we see that $\log ^{+}|F(x)|$ will not belong to $B_{1}$ if

$$
\sum \frac{J_{n}}{x_{n}^{2}} \log \frac{x_{n}}{\sqrt{J_{n}}}=\infty .
$$


The choice $J_{n} / x_{n}^{2}=n^{-1}(\log n)^{-2}$ fulfills all conditions.

Upon re-examining the proof and referring to the remarks following Theorem 12, we obtain:

EXAMPLE 5. Let $\left\{I_{n}\right\}$ be a given sequence of positive numbers such that

$$
I_{n} \rightarrow 0, \quad \sum I_{n} \log \frac{1}{I_{n}}<\infty
$$

Let $\left\{x_{n}\right\}$ be a given geometric sequence with ratio $>1$. If

$$
\int_{x_{n}}^{x_{n+1}} \frac{\Lambda(x)}{x^{2}} d x \leqq I_{n}
$$

then the corresponding even entire function $F$ satisfies $\log ^{+}|F(x)| \in B_{1}$. But if the series diverges there is an even function $F$ of this sort, with integral zeros, such that $\log ^{+}|F(x)|$ is not in $B_{1}$.

A result related to Theorem 11 is:

EXAMPLE 6. Let $h(r)$ be an increasing function satisfying

$$
\int_{-r}^{r} \Lambda(u) d u \leqq h^{2}(r) .
$$

If $h(r) \in B_{1}$, then $\log ^{+}|F(x)| \in B_{1}$ holds for the even canonical product, $F$, formed from the $\lambda_{n}{ }^{\prime} s$. But if $h$ is not in $B_{1}$ there is such a function $F$, with $A$ satisfying the above inequality and with $\log ^{+}|F(x)| \operatorname{not}$ in $B_{1}^{+}$.

The proof is left to the reader.

Note added October 1966.

According to a recent unpublished result of Matzayev, $\Lambda(u)-$ $D u \in B_{1}^{+}$implies $\log |F(x)| \in B_{1}$ for even entire functions of genus 1 . Hence, the condition $\Lambda \in B_{1}$ in Example 4 cannot be replaced by $\Lambda \in B_{1}^{+}$. Actually, Theorem 10 yields the following generalization of Matzayev's theorem to meromorphic functions which need not be even:

$$
\Lambda(u) \in B_{1}^{+} \Rightarrow \log |F(x)|-\widetilde{\Lambda}(x) \in B_{1} .
$$

Because of the interest and simplicity of this result, we present an independent development of it elsewhere.

\section{BIBLIOGRAPHY}

1. A. Beurling, and P. Malliavin, On Fourier transforms of measures with compact support, Acta Math. 105 (1961), 141-175.

2. R. P. Boas, Jr., Entire functions, New York, 1954. References to the original 
sources are given here.

3. - The growth of analytic functions on a line, J. Analyse Math. 4 (1955), 1-28. 4. J. P. Kahane, Sur la totalité des suites d'exponentielles imaginaires, Ann. Inst. Fourier 8 (1959), 273-275.

5. J. P. Kahane, and L. A. Rubel, On Weierstrass products of zero type on the real axis, Illinois J. Math. 4 (1960), 584-592.

6. J. Korevaar, T. van Aardenne-Ehrenfest, and N. G. de Bruijn, $A$ note on slowly oscillating functions, Nieuw Arch. Wisk. (2) 23 (1949), 77-86.

7. Paul Koosis, Sur la totalité des systèmes d'exponentielles imaginaires, Comptes Rendus 250 (1960), 2102-2103.

8. - Sur la non-totalité de certaines suites d'exponentielles sur des intervalles assez longues, Ann. Sci. École Norm. Sup. (2) 75 (1958), 125-152.

9. B. Levin, Jr., Distribution of zeros of entire functions, Amer. Math. Soc. Trans., Providence, R.I.

10. S. Mandelbrojt, Transformée de Fourier de fonctions entières et séries de Dirichlet: un principe de dualité, J. Analyse Math. 10 (1963), 381-404.

11. R. M. Redheffer, On even entire functions with zeros having a density, Trans. Amer. Math. Soc. 77 (1954), 32-61.

12. - Ganze Funktionen und Vollständigkeit, Österreich. Akad. Wiss. 6 (1957), 96-99.

Received February 8, 1966. The preparation of this paper was sponsored by the Office of Naval Research. Reproduction in whole or in part is permitted for any purpose of the United States Government.

University of CALIFornia, Los ANgeles 


\title{
PACIFIC JOURNAL OF MATHEMATICS
}

\author{
EDITORS
}

\section{H. ROYDEN}

Stanford University

Stanford, California

\section{J. P. JANS}

University of Washington Seattle, Washington 98105

\section{J. DugundJI}

Department of Mathematics

Rice University

Houston, Texas 77001

RICHARD ARENS

University of California

Los Angeles, California 90024

\section{ASSOCIATE EDITORS}

E. F. BECKENBACH
F. WOLF

K. YosidA

\section{SUPPORTING INSTITUTIONS}

\author{
UNIVERSITY OF BRITISH COLUMBIA \\ CALIFORNIA INSTITUTE OF TECHNOLOGY \\ UNIVERSITY OF CALIFORNIA \\ MONTANA STATE UNIVERSITY \\ UNIVERSITY OF NEVADA \\ NEW MEXICO STATE UNIVERSITY \\ OREGON STATE UNIVERSITY \\ UNIVERSITY OF OREGON \\ OSAKA UNIVERSITY \\ UNIVERSITY OF SOUTHERN CALIFORNIA
}

\author{
STANFORD UNIVERSITY \\ UNIVERSITY OF TOKYO \\ UNIVERSITY OF UTAH \\ WASHINGTON STATE UNIVERSITY \\ UNIVERSITY OF WASHINGTON \\ MERICAN MATHEMATICAL SOCIETY \\ CHEVRON RESEARCH CORPORATION \\ TRW SYSTEMS \\ NAVAL ORDNANCE TEST STATION
}

Mathematical papers intended for publication in the Pacific Journal of Mathematics should be in typed form or offset-reproduced, double spaced with large margins. Underline Greek letters in red, German in green, and script in blue. The first paragraph or two must be capable of being used separately as a synopsis of the entire paper. It should not contain references to the bibliography. Manuscripts may be sent to any one of the four editors. All other communications to the editors should be addressed to the managing editor, Richard Arens, University of California, Los Angeles, California 90024.

Each author of each article receives 50 reprints free of charge; additional copies may be obtained at cost in multiples of 50 .

The Pacific Journal of Mathematics is published monthly. Effective with Volume 16 the price per volume (3 numbers) is $\$ 8.00$; single issues, $\$ 3.00$. Special price for current issues to individual faculty members of supporting institutions and to individual members of the American Mathematical Society: $\$ 4.00$ per volume; single issues $\$ 1.50$. Back numbers are available.

Subscriptions, orders for back numbers, and changes of address should be sent to Pacific Journal of Mathematics, 103 Highland Boulevard, Berkeley 8, California.

Printed at Kokusai Bunken Insatsusha (International Academic Printing Co., Ltd.), 7-17, Fujimi 2-chome, Chiyoda-ku, Tokyo, Japan.

PUBLISHED BY PACIFIC JOURNAL OF MATHEMATICS, A NON-PROFIT CORPORATION

The Supporting Institutions listed above contribute to the cost of publication of this Journal, but they are not owners of publishers and have no responsibility for its content or policies. 


\section{Pacific Journal of Mathematics}

\section{Vol. 24, No. $2 \quad$ June, 1968}

John Suemper Alin and Spencer Ernest Dickson, Goldie's torsion theory and its derived functor . . . . . . . . . . . . . . . . . . . . . . . . . . . 195

Steve Armentrout, Lloyd Lesley Lininger and Donald Vern Meyer,

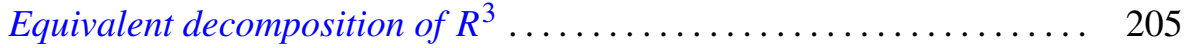

James Harvey Carruth, A note on partially ordered compacta ........... 229

Charles E. Clark and Carl Eberhart, A characterization of compact

connected planar lattices........................... 233

Allan Clark and Larry Smith, The rational homotopy of a wedge ......... 241

Donald Brooks Coleman, Semigroup algebras that are group algebras .... 247

John Eric Gilbert, Convolution operators on $L^{p}(G)$ and properties of

locally compact groups ............................. 257

Fletcher Gross, Groups admitting a fixed-point-free automorphism of order

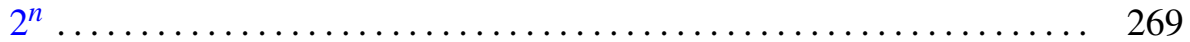

Jack Hardy and Howard E. Lacey, Extensions of regular Borel measures . . . 277

R. G. Huffstutler and Frederick Max Stein, The approximation solution of

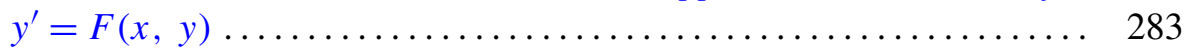

Michael Joseph Kascic, Jr., Polynomials in linear relations . . . . . . . . . . 291

Alan G. Konheim and Benjamin Weiss, A note on functions which

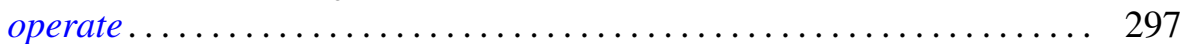

Warren Simms Loud, Self-adjoint multi-point boundary value problems ... 303 Kenneth Derwood Magill, Jr., Topological spaces determined by left ideals

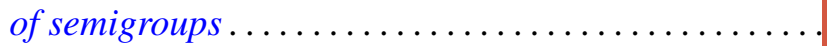

Morris Marden, On the derivative of canonical products . . .

J. L. Nelson, A stability theorem for a third order nonlinear differential

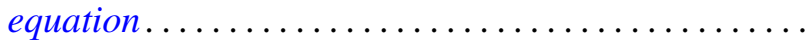

Raymond Moos Redheffer, Functions with real poles and zeros ...

Donald Zane Spicer, Group algebras of vector-valued functions ...

Myles Tierney, Some applications of a property of the functor $E f$ 